\title{
Exploring CP-violation, via heavy neutrino oscillations, in rare $B$ meson decays at Belle II
}

\author{
Gorazd Cvetič $^{1, \mathrm{a}}$, C. S. Kim ${ }^{2, \mathrm{~b}}$, Sebastian Mendizabal ${ }^{1, \mathrm{c}}$, Jilberto Zamora-Saá ${ }^{3, \mathrm{~d}}$ \\ ${ }^{1}$ Department of Physics, Universidad Técnica Federico Santa María, Valparaíso, Chile \\ ${ }^{2}$ Department of Physics and IPAP, Yonsei University, Seoul 03722, Korea \\ ${ }^{3}$ Departamento de Ciencias Físicas, Universidad Andres Bello, Sazié 2212, Piso 7, Santiago, Chile
}

Received: 8 July 2020 / Accepted: 2 November 2020 / Published online: 16 November 2020

(C) The Author(s) 2020

\begin{abstract}
In this article we study the rare B-meson decay via two on-shell almost-degenerate Majorana Heavy Neutrinos, into two charged leptons and two pseudoscalar mesons $\left(B^{ \pm} \rightarrow D^{0} \ell_{1}^{ \pm} \ell_{2}^{ \pm} \pi^{\mp}\right)$. We consider the scenario where the heavy neutrino masses are $\sim 2 \mathrm{GeV}$ and the heavy-light mixing coefficients are $\left|B_{\ell N}\right|^{2} \sim 10^{-5}$, and evaluate the possibility to measure the CP-asymmetry at Belle II. We present some realistic conditions under which the asymmetry could be detected.
\end{abstract}

\section{Introduction}

The first indications of physics beyond the Standard Model (SM) come from: neutrino oscillations (NOs), baryonic asymmetry of the Universe (BAU) and dark matter (DM). During the last years NOs experiments have confirmed that active neutrinos $(v)$ are very light massive particles $[1,2]$ and consequently the SM must be extended. The evidence of neutrino masses that arises from oscillations were first predicted in [3] and later observed in [1,4-7]. These extremely light masses can be explained with the introduction of sterile neutrinos and via the seesaw mechanism [8-10]. The outcome gives us Majorana neutrinos with light eigenstates $m_{v} \lesssim 1$ $\mathrm{eV}$ and heavy neutrino $(\mathrm{HN})$ eigenstates. The masses of the $\mathrm{HN}$ particles are normally taken in the $M_{N} \gg 1 \mathrm{TeV}$ regime. However, there are other seesaw scenarios with lower masses for the HN, $M_{N} \sim 1 \mathrm{TeV}$ [11-17] and $M_{N} \sim 1 \mathrm{GeV}$ [1826]. If one goes to $\mathrm{HN}$ mass scales of the order of the light neutrinos, new contributions to the seesaw neutrino masses should be taken into account (see for example [27]). Prob-

\footnotetext{
a e-mail: gorazd.cvetic@usm.cl

be-mail: cskim@yonsei.ac.kr

c e-mail: sebastian.mendizabal@usm.cl

de-mail: jilberto.zamora@unab.cl (corresponding author)
}

ing the nature of neutrinos has been one of the most interesting and elusive tasks in modern physics. Experimentally, whether they are Dirac or Majorana fermions can be, in principle, established in neutrinoless double beta decay $(0 v \beta \beta)$ experiments [28-36], rare lepton number violating (LNV) decays of mesons [37-55] and of $\tau$ lepton [56-60], and specific scattering processes $[19,61-83]$.

The nature of Dirac neutrinos only allows them to appear in processes that are lepton number conserving (LNC). Majorana neutrinos can induce both lepton number conserving and lepton number violating (LNV) processes, which allows a wider spectrum of physics to take place. An important example of this is baryogenesis via leptogenesis, where the $\mathrm{LNV}$ and CP-violating processes can lead to a generation of a lepton number asymmetry in the early universe, which is then converted (through sphaleron processes $[10,84,85]$ ) to the baryon number asymmetry observed in the universe [86]. There are many different models that try to explain this asymmetry. However, two standard approaches that use Majorana neutrinos for successful Leptogenesis are out-of-equilibrium $H N$ decays (or Thermal Leptogenesis) and leptogenesis from oscillations. Both of them use sterile neutrinos as an extension to the standard model, with their masses being calculated with the seesaw type-I mechanism. This mechanism allows us to have heavy neutrinos using the fact that the SM neutrinos have very low masses. These HNs satisfy the Sakharov conditions [87] in order to produce the asymmetry dynamically. Consequently, thermal leptogenesis [88-90] takes into account the lepton number asymmetry generated by the decay of a massive Majorana neutrino in a thermal bath, while the latter, known as Akhmedov-Rubakov-Smirnov (ARS) mechanism [91], leads to a lepton number asymmetry by means of $\mathrm{HN}$ oscillations. The main difference between the two mechanisms comes from the fact that the first case is a freeze-out situation while the ARS mechanism can be seen as a freeze-in one. 
The range of the $\mathrm{HN}$ masses for thermal leptogenesis is dictated by the amount of CP violation that can be generated ${ }^{1}$. In the most simple scenarios leptogenesis is constructed with masses $M_{N} \gtrsim 10^{10} \mathrm{GeV}$, or $M_{N} \gtrsim 1 \mathrm{TeV}$ if one takes into account resonant effects [93], whereas the ARS mechanism allows neutrinos to reach masses as low as $\sim 1 \mathrm{GeV}$. The $\mathrm{HN}$ mass scale for thermal leptogenesis cannot be reached in modern experiments, while ARS leptogenesis allows a variety of experiments to try and probe not only the nature of neutrinos, but also leptogenesis [94].

The search for the $\mathrm{CP}$ violation has been studied in different scenarios: resonant (overlap) scattering processes [9597], resonant leptonic [98-100] and semileptonic rare meson decays [99,101,102], as well as $B$ mesons, $W$ bosons and $\tau$ decays that include heavy neutrinos oscillation $[60,103-$ 109]. The resonant (overlap) effect comes from the interference between two almost degenerate neutrino mass eigenstates with masses of order $\sim \mathrm{GeV}$.

This article is organized in the following way: In Sect. 2 we present the effective CP-violating $B$ meson decay width for the LNV process $B^{ \pm} \rightarrow D^{0} \ell_{1}^{ \pm} \ell_{2}^{ \pm} \pi^{\mp}$, and in Appendices A$\mathrm{D}$ more details are given. In Sect. 3 we present the numerical results for this effective branching ratio (with $\ell_{1}=\ell_{2}=\mu$ ) and for the related $\mathrm{CP}$ asymmetry ratio, for different values of the detector length, of the ratio of the HN mass difference and the HN total decay width, and for different values of the CP-violating phase. In Sect. 4 we discuss the possibility for the detection of various such signals within the detector at Belle II and summarize our results.

\section{CP violation in heavy neutrino decay}

The simplest extension of the SM that explains the smallness of the active neutrino masses is the addition of righthanded neutrinos $\left(v_{R}\right)$. Then, the relevant terms of the new Lagrangian $\mathcal{L}_{N}$ will read

$-\mathcal{L}_{N}=Y_{\nu} \bar{\ell}_{L} \phi v_{R}+\frac{M_{R}}{2} \overline{\left(v_{R}\right)^{c}} v_{R}+$ h.c.,

where $M_{R}$ is the mass of the right-handed neutrinos. After diagonalizing the mass matrix, three very light neutrinos are obtained, as well as three heavy ones, this is the well known seesaw mechanism [8-10]. The mass of the light neutrinos will be given by

$m_{v} \propto\langle\phi\rangle^{2} \frac{Y_{v} Y_{v}^{T}}{M_{N}}$,

${ }^{1}$ In the type-I seesaw mechanism, the mass scale was first discussed in [92] and it is known as the Davidson-Ibarra bound. where $M_{N}=M_{R}$ is a $3 \times 3$ mass matrix of the heavy neutrinos and $\langle\phi\rangle$ is the electroweak vacuum expectation value of the Higgs field. By tuning the parameters in the above equation one can reach neutrino masses $\sim 1 \mathrm{GeV}$, resulting in Yukawa couplings $\sim 10^{-5}$. This type of scenario is well discussed in the $v$ MSM model [20,21]. Two key ingredients in this model are the $\mathrm{CP}$ violation that occurs in the mixing of the heavy neutrinos and a resonant effect when the masses of two of them satisfy the condition $\Delta M_{N}\left(\equiv M_{N_{2}}-M_{N_{1}}\right)$ $=\Gamma_{N}$.

In previous articles we explored the HN CP-violating decays: $i$ ) considering only resonant $\mathrm{CP}$ violation without HN oscillation effects [98,100,101,106] and $i i)$ nonresonant HN oscillation effects [60,103-105]. In this article, we will considerer the decay $B^{ \pm} \rightarrow D^{0} \ell_{1}^{ \pm} \ell_{2}^{ \pm} \pi^{\mp}$ (see Fig. 1) extending the previous analysis, by considering simultaneously both of the aforementioned CP-violating sources, in order to explore these signals at Belle II experiment.

In this work we will assume the existence of several (three) Heavy Neutrino states $N_{j}(j=1,2,3)$, with respective masses $M_{N_{j}}$. In addition, we will assume that the first two heavy neutrinos are almost degenerate and with masses in the range of $\sim 1 \mathrm{GeV}$, and the third neutrino is much heavier

$M_{N_{3}} \gg M_{N_{2}} \approx M_{N_{1}} \sim 1 \mathrm{GeV} \quad\left(M_{N_{2}}>M_{N_{1}}\right)$.

The first three active neutrinos $\nu_{\ell}$ (where $\ell=e, \mu, \tau$ ) will have, in general, admixtures of the above mentioned heavy mass eigenstates

$v_{\ell}=\sum_{j=1}^{3} B_{\ell j} v_{j}+B_{\ell N_{1}} N_{1}+B_{\ell N_{2}} N_{2}+B_{\ell N_{3}} N_{3}$

where the heavy-light mixing elements $B_{\ell N_{j}}$ are, in general, small complex numbers

$B_{\ell_{k} N_{j}} \equiv\left|B_{\ell_{k} N_{j}}\right| e^{i \phi_{k j}}, \quad(k, j=1,2,3)$

We will consider CP-violating decays of $B$ mesons into two light leptons $\left(\ell_{1} \ell_{2}\right)$ and a pion, mediated by heavy onshell neutrinos $N_{j}(j=1,2)$. It turns out that (effective) branching ratios for the decays of the type $B \rightarrow D \ell_{1} \ell_{2} \pi$ (cf. Fig. 1) are significantly larger than the decays $B \rightarrow$ $\ell_{1} \ell_{2} \pi$, by about a factor of $30-40$ when $M_{N} \approx 2 \mathrm{GeV}$, cf. Ref. [110] (Figs. 19a and 20a there), ${ }^{2}$ the main reason been the different CKM matrix elements $\left|V_{c b}\right| \sim 10\left|V_{u b}\right|$. For this reason, we will consider the decay channels $B \rightarrow D \ell_{1} \ell_{2} \pi$ (Fig. 1). The heavy neutrino $N_{3}$ will not enter our considerations because, in contrast to $N_{1}$ and $N_{2}$, it is off-shell

\footnotetext{
${ }^{2}$ Majorana neutrinos in $B$ meson decays were considered also in Refs. [111-113].
} 
Fig. 1 Feynmann diagram of the decay processes

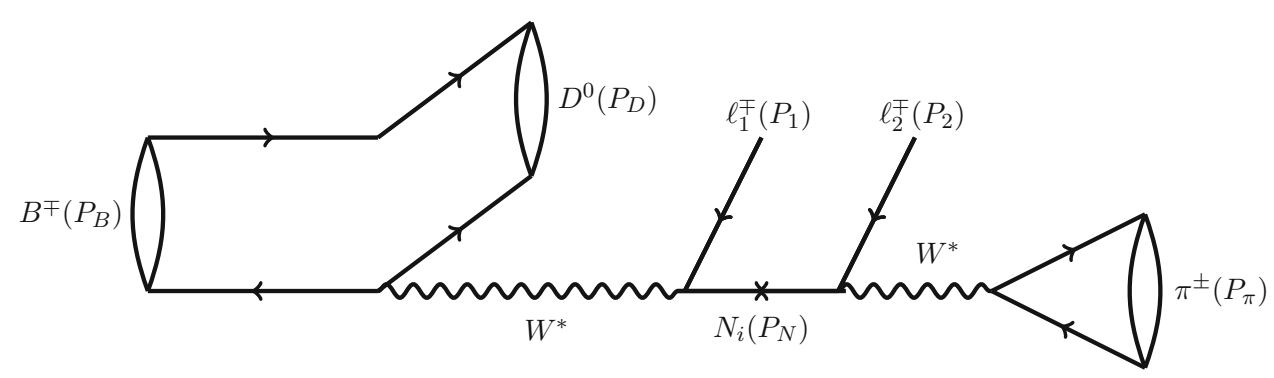

in these decays. Furthermore, in order to avoid the kinematic suppression from heavy leptons, we exclude from our consideration the case of $\tau$-lepton production. In addition, to avoid the present stringent upper bounds on the heavylight mixing $B_{e N_{j}}$, we also exclude from our consideration the case of $\ell=e$ lepton production. Thus, we will take $\ell_{1} \ell_{2}=\mu \mu$. The $N_{1}-N_{2}$ oscillation effects in such decays $\left(\ell_{1} \ell_{2}=\mu \mu\right)$ turn out to disappear in LNC decays but survive in LNV decays [103]. Hence, we will consider the LNV decays $B^{ \pm} \rightarrow D^{0} \mu^{ \pm} \mu^{ \pm} \pi^{\mp}$ (Fig. 1). The CP-violating $B$ meson decay width for such a process, which accounts for the fact that the process will be detected only if the HN decays during its crossing through the detector (effective $\Gamma$ ), and includes both the overlap (resonant) $[98,101]$ and the HNoscillation CP-violating sources [103-105], is given by

$$
\begin{aligned}
\Gamma_{\text {eff }} & \left(B^{ \pm} \rightarrow D^{0} \ell_{1}^{ \pm} \ell_{2}^{ \pm} \pi^{\mp}\right) \\
= & \bar{\Gamma}\left(B^{ \pm} \rightarrow D^{0} \ell_{1}^{ \pm} N\right) \bar{\Gamma}\left(N \rightarrow \ell_{2}^{ \pm} \pi^{\mp}\right) 2\left|B_{\ell_{1} N}\right|^{2}\left|B_{\ell_{2} N}\right|^{2} \\
& \times\left[\frac { 1 } { \Gamma _ { N } } ( 1 - \operatorname { e x p } ( - \frac { - L \Gamma _ { N } } { \gamma _ { N } ^ { \prime \prime } \beta _ { N } ^ { \prime \prime } } ) ) \left(1+\delta(Y) \cos \left(\theta_{L V}\right)\right.\right. \\
& \left.\mp \frac{\eta(Y)}{Y} \sin \left(\theta_{L V}\right)\right)+\frac{1}{\Gamma_{N}\left(1+Y^{2}\right)} \\
& \times\left\{\operatorname { e x p } ( - \frac { - L \Gamma _ { N } } { \gamma _ { N } ^ { \prime \prime } \beta _ { N } ^ { \prime \prime } } ) \left[Y \sin \left(\frac{2 \pi L}{L_{\mathrm{osc}}} \pm \theta_{L V}\right)\right.\right. \\
& \left.-\cos \left(\frac{2 \pi L}{L_{\mathrm{osc}}} \pm \theta_{L V}\right)\right] \\
+ & \left.\left.\left(\cos \left(\theta_{L V}\right) \mp Y \sin \left(\theta_{L V}\right)\right)\right\}\right]
\end{aligned}
$$

where $L$ stands for the distance (in the lab frame) between the two vertices of the process (the flight length of the onshell neutrino $\left.N_{j}\right),{ }^{3} L_{\mathrm{osc}}=\left(2 \pi \gamma_{N}^{\prime \prime} \beta_{N}^{\prime \prime}\right) / \Delta M_{N}$ is the $\mathrm{HN}$ oscillation length,

$Y \equiv \frac{\Delta M_{N}}{\Gamma_{N}}, \quad \Delta M_{N} \equiv M_{N_{2}}-M_{N_{1}}(>0)$,

\footnotetext{
${ }^{3} L$ is thus limited by the (effective) length $L_{\text {det }}$ of the detector, $L \leq L_{\text {det }}$. The lab frame in this work is denoted by $\Sigma^{\prime \prime}$. However, for simplicity of notation, the distance $L^{\prime \prime}$ in the lab frame will be denoted simply as $L$.
}

and $\theta_{L V}$ is the CP-violating phase ${ }^{4}$ which, according to the notation of Eq. (5) can be written as

$\theta_{L V} \equiv \theta_{k j}=\left(\phi_{1 k}+\phi_{2 k}-\phi_{1 j}-\phi_{2 j}\right), \quad(k, j=1,2)$

Further, the functions $\eta(Y)$ and $\delta(Y)$ are $[98,100,101]$

$\eta(Y)=\frac{Y^{2}}{Y^{2}+1}, \quad \delta(Y)=\frac{1}{Y^{2}+1}$.

The numerical values of $\eta(Y)$ and $\delta(Y)$ were obtained in [98,101], and the explicit expression for $\eta(Y)$ was obtained in [100] (App. 6 there). Based on the mentioned numerical values of $\delta(Y)$ (cf. Table I in [101], Table II in [98], and Table 4 in [100]), we observe a posteriori here that they can be reproduced with high precision by the explicit expression for $\delta(Y)$ given here. The functions $\delta(Y)$ and $\eta(Y)$ are related with the real and imaginary parts, respectively, of the product of scattering amplitudes for the processes $W^{*} \rightarrow \ell_{1} N_{j} \rightarrow \ldots$ $(j=1,2)$, and they involve the product of (almost on-shell) propagators of the nearly degenerate neutrinos $N_{1}$ and $N_{2}$. We refer for details to Refs. [98,100,101].

In Eq. (6), the HN Lorentz kinematical parameters in the lab frame $\left(\Sigma^{\prime \prime}\right) \beta_{N}^{\prime \prime}$ and $\gamma_{N}^{\prime \prime}=1 / \sqrt{1-\left(\beta_{N}^{\prime \prime}\right)^{2}}$ are assumed to be constant. This can be extended to the realistic case of variable $\beta_{N}^{\prime \prime}$ [111], and this extension is explained in Appendix C. We also assumed that $\left|B_{\ell N_{1}}\right|=\left|B_{\ell N_{2}}\right|\left(\equiv\left|B_{\ell N}\right|\right)$, with $\ell=\mu, e, \tau$.

Furthermore, the expression (6), in addition to the aforementioned approximations (fixed $\beta_{N}^{\prime \prime}$ and common $\left|B_{\ell N}\right|$ 's), is obtained in an approximation of combining the overlap (resonant) and oscillation effects, which is valid when $Y$ is significantly larger than one, e.g. $Y \gtrsim 5$. This is explained in more detail in Appendix D, where several steps of derivation of the expression (6) are given.

In general, $\Gamma_{N}=\left(\Gamma_{N_{1}}+\Gamma_{N_{2}}\right) / 2$ where $\Gamma_{N_{j}}$ is the total decay width of $\mathrm{HN} N_{j}(j=1,2)$. However, due to our assumption $\left|B_{\ell N_{1}}\right|=\left|B_{\ell N_{2}}\right|\left(\equiv\left|B_{\ell N}\right|\right)$, we have $\Gamma_{N_{1}}=$

\footnotetext{
$\overline{{ }^{4} \text { For example, }}$ if $\ell_{1}=\ell_{2}=\mu$, then $\theta_{L V} \equiv \theta_{21}=2\left(\phi_{\mu 2}-\phi_{\mu 1}\right)=$ $2\left[\arg \left(B_{\mu N_{2}}\right)-\arg \left(B_{\mu N_{1}}\right)\right]$.
} 


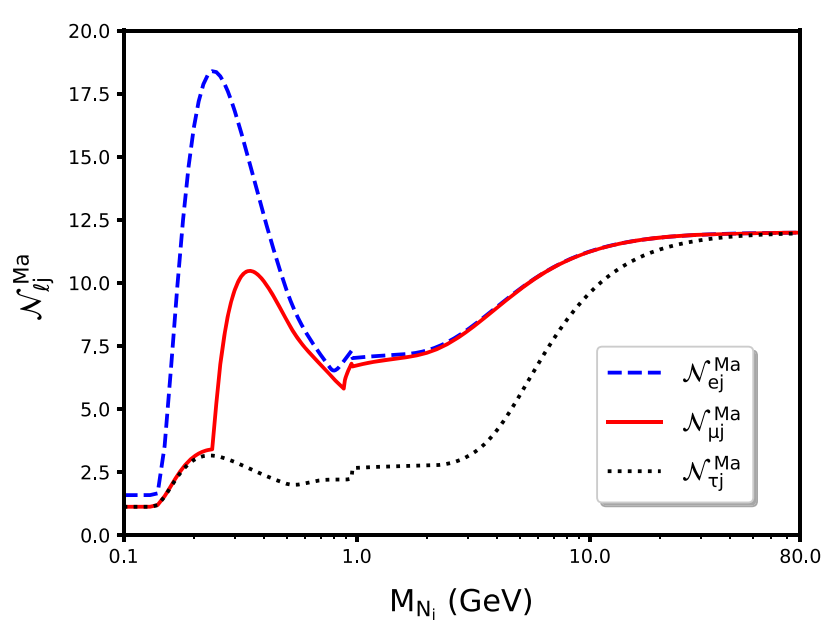

Fig. 2 Effective mixing coefficients $\mathcal{N}_{\ell j}^{\mathrm{Ma}}$ for Majorana neutrinos

$\Gamma_{N_{2}}=\Gamma_{N}$. This is because the total decay width of the heavy neutrino $N_{j}$ is $[98,100]$

$$
\begin{aligned}
& \Gamma_{N_{j}} \approx \mathcal{K}_{j}^{\mathrm{Ma}} \frac{G_{F}^{2} M_{N_{j}}^{5}}{96 \pi^{3}}, \quad \mathcal{K}_{i}^{\mathrm{Ma}} \\
& \quad=\mathcal{N}_{e j}^{\mathrm{Ma}}\left|B_{e N_{j}}\right|^{2}+\mathcal{N}_{\mu j}^{\mathrm{Ma}}\left|B_{\mu N_{j}}\right|^{2}+\mathcal{N}_{\tau j}^{\mathrm{Ma}}\left|B_{\tau N_{j}}\right|^{2},
\end{aligned}
$$

where $\mathcal{N}_{\ell j}^{\mathrm{Ma}}$ are the effective mixing coefficients whose range is $\sim 1-10$ and account for all possible $\mathrm{HN}$ decay channels. The $N_{j}$ coefficients are presented in Fig. 2.

From now on, as mentioned earlier we will consider only the case $\ell_{1}=\ell_{2}=\mu$. We notice that $\left|B_{\mu N_{j}}\right|^{2} \approx\left|B_{\tau N_{j}}\right|^{2} \lesssim$ $10^{-5}$ and $\left|B_{e N_{j}}\right|^{2}<10^{-7}$, so that the $\mathcal{K}_{j}^{\mathrm{Ma}}$ can receive significant contribution only from $\mu$ and $\tau$ decay channels (note that $\mathcal{N}_{\mu j}^{\mathrm{Ma}}+\mathcal{N}_{\tau j}^{\mathrm{Ma}} \approx 10$ ). The mixings $B_{\mu N_{1}}$ and $B_{\mu N_{2}}$ can be, in principle, significantly different for the two HNs, and therefore, the two mixing factors $\mathcal{K}_{j}^{\mathrm{Ma}}(j=1,2)$ may differ significantly from each other. However, as mentioned earlier, in this work we will assume that $\left|B_{\ell N_{1}}\right|=\left|B_{\ell N_{2}}\right|\left(\equiv\left|B_{\ell N}\right|\right)$. Taking $\mathcal{K}_{1}^{\mathrm{Ma}} \approx \mathcal{K}_{2}^{\mathrm{Ma}}=10\left|B_{\mu N}\right|^{2}$ the $\mathrm{HN}$ total decay width then reads

$\Gamma_{N}\left(M_{N}\right)=10\left|B_{\mu N}\right|^{2} \frac{G_{F}^{2} M_{N}^{5}}{96 \pi^{3}}$,

we also note that the $\mathrm{HN}$ masses are almost equal, i.e. $M_{N_{j}} \simeq$ $M_{N}$.

The usual measure of the relative $\mathrm{CP}$ violation effect is given by the $\mathrm{CP}$ asymmetry ratio

$A_{\mathrm{CP}}=\frac{\Gamma_{\mathrm{eff}}\left(B^{+} \rightarrow D^{0} \mu^{+} \mu^{+} \pi^{-}\right)-\Gamma_{\mathrm{eff}}\left(B^{-} \rightarrow D^{0} \mu^{-} \mu^{-} \pi^{+}\right)}{\Gamma_{\mathrm{eff}}\left(B^{+} \rightarrow D^{0} \mu^{+} \mu^{+} \pi^{-}\right)+\Gamma_{\mathrm{eff}}\left(B^{-} \rightarrow D^{0} \mu^{-} \mu^{-} \pi^{+}\right)}$.

\section{Results}

In this section we show the numerical results for the effective branching ratio $\mathrm{Br}_{\text {eff }}\left(\mathrm{B}^{ \pm}\right)=\Gamma_{\text {eff }}\left(B^{ \pm} \rightarrow D^{0} \mu^{ \pm} \mu^{ \pm} \pi^{\mp}\right) / \Gamma$ $\left(B \rightarrow\right.$ all) and the $\mathrm{CP}$ asymmetry ratio $\mathrm{A}_{\mathrm{CP}}$ in (12) for different values of the $Y$ parameter and the maximal displaced vertex length $L$, which can be interpreted as the (effective) detector length ( $L \leq L_{\text {det }}$ ). The calculations were performed by numerical integration with the VEGAS algorithm [114] in each step of $L$ and $Y$. All integrations were performed using $M_{N}=2 \mathrm{GeV}$ and heavy-light mixings $\left|B_{\mu N}\right|^{2}=\left|B_{\tau N}\right|^{2}=10^{-5}$. The selected mixing values are consistent with the present experimental constraints given in Refs. [48, 115] and references therein. Moreover, two different values (scenarios) were chosen for the CP-violating phase: $\theta_{L V}=\pi / 2, \pi / 4$.

The kinematical Lorentz factor $\gamma_{N}^{\prime \prime}$ and $\beta_{N}^{\prime \prime}$ in Eq. (6) in reality are not fixed, but vary and are obtained as explained in Appendix C [Eq. (C3)], where the general expression $\Gamma_{\text {eff }}$ for the case of only one $\mathrm{HN} N$ is given in Eq. (C4). In the case of two (almost degenerate) HNs $N_{j}(j=1,2)$ the expression (C4) gets extended by the overlap (resonant) and oscillation terms as those appearing in Eq.(6), leading to our main formula

$$
\begin{aligned}
\Gamma_{\text {eff }} & \left(B^{ \pm} \rightarrow D^{0} \ell_{1}^{ \pm} \ell_{2}^{ \pm} \pi^{\mp}\right) \\
= & 2\left|B_{\ell_{1} N}\right|^{2}\left|B_{\ell_{2} N}\right|^{2} \frac{\bar{\Gamma}\left(N \rightarrow \ell_{2} \pi\right)}{\Gamma_{N}} \int d q^{2} \int d \Omega_{\hat{q}^{\prime}} \\
& \times \int d \Omega_{\hat{p}_{1}} \frac{d \bar{\Gamma}\left(B \rightarrow D \ell_{1} N\right)}{d q^{2} d \Omega_{\hat{q}^{\prime}} d \Omega_{\hat{p}_{1}}} \\
& \times\left[\left\{1-\exp \left(-L \Gamma_{N} / \sqrt{\left.\left(E_{N}^{\prime \prime}\left(q^{2} ; \hat{q}^{\prime}, \hat{p}_{1}\right) / M_{N}\right)^{2}-1\right)}\right\}\right.\right. \\
& \times\left[1+\delta(Y) \cos \left(\theta_{L V}\right) \mp \frac{\eta(Y)}{Y} \sin \left(\theta_{L V}\right)\right] \\
& +\frac{1}{\left(1+Y^{2}\right)}\left\{\exp \left(-L \Gamma_{N} / \sqrt{\left(E_{N}^{\prime \prime}\left(q^{2} ; \hat{q}^{\prime}, \hat{p}_{1}\right) / M_{N}\right)^{2}-1}\right)\right. \\
& \times\left[Y \sin \left(\frac{2 \pi L}{L_{\mathrm{osc}}} \pm \theta_{L V}\right)-\cos \left(\frac{2 \pi L}{L_{\mathrm{osc}}} \pm \theta_{L V}\right)\right] \\
& \left.\left.+\left(\cos \left(\theta_{L V}\right) \mp Y \sin \left(\theta_{L V}\right)\right)\right\}\right] .
\end{aligned}
$$

Here we should keep in mind that the oscillation length $L_{\mathrm{osc}}$ relies on the (variable) Lorentz factors $\beta_{N}^{\prime \prime}$ and $\gamma_{N}^{\prime \prime}$, namely $2 \pi / L_{\text {osc }}=Y \Gamma_{N} /\left(\gamma_{N}^{\prime \prime} \beta_{N}^{\prime \prime}\right)$ [cf. Eq. (D4)], so it also depends on the integration variables $q^{2}, \hat{q}^{\prime}$ and $\hat{p}_{1}$ via $E_{N}^{\prime \prime}\left(q^{2} ; \hat{q}^{\prime}, \hat{p}_{1}\right)$, cf. Eq. (C3). ${ }^{5}$

In order to evaluate the relevance of Oscillatory and Overlapping effects on the main decay channel, we can either: (a)

\footnotetext{
${ }^{5}$ From the expression (13), and using Eq. (9), it can be checked after some algebra that in the limit $Y=0$ the two decay widths (i.e., for $B^{+}$ and $B^{-}$) become equal to each other.
} 
disregard in Eq. (13) the overlap (resonant) terms and include only the oscillatory terms

$$
\begin{aligned}
\Gamma_{\text {eff }}^{\text {osc }} & \left(B^{ \pm} \rightarrow D^{0} \ell_{1}^{ \pm} \ell_{2}^{ \pm} \pi^{\mp}\right) \\
= & 2\left|B \ell_{1} N\right|^{2}\left|B_{\ell_{2} N}\right|^{2} \frac{\Gamma\left(N \rightarrow \ell_{2} \pi\right)}{\Gamma_{N}} \int d q^{2} \\
& \times \int d \Omega_{\hat{q}^{\prime}} \int d \Omega_{\hat{p}_{1}} \frac{d \bar{\Gamma}\left(B \rightarrow D \ell_{1} N\right)}{d q^{2} d \Omega_{\hat{q}^{\prime}} d \Omega_{\hat{p}_{1}}} \\
& \times\left[\left\{1-\exp \left(-L \Gamma_{N} / \sqrt{\left(E_{N}^{\prime \prime}\left(q^{2} ; \hat{q}^{\prime}, \hat{p}_{1}\right) / M_{N}\right)^{2}-1}\right)\right\}\right. \\
& +\frac{1}{\left(1+Y^{2}\right)}\left\{\exp \left(-L \Gamma_{N} / \sqrt{\left(E_{N}^{\prime \prime}\left(q^{2} ; \hat{q}^{\prime}, \hat{p}_{1}\right) / M_{N}\right)^{2}-1}\right)\right. \\
& \times\left[Y \sin \left(\frac{2 \pi L}{L_{\mathrm{osc}}} \pm \theta_{L V}\right)\right. \\
& \left.-\cos \left(\frac{2 \pi L}{L_{\mathrm{osc}}} \pm \theta_{L V}\right)\right] \\
& \left.\left.+\left(\cos \left(\theta_{L V}\right) \mp Y \sin \left(\theta_{L V}\right)\right)\right\}\right]
\end{aligned}
$$

that $\exp \left(-L \Gamma_{N} /\left(\gamma_{N}^{\prime \prime} \beta_{N}^{\prime \prime}\right)\right)$ is almost zero, and consequently the oscillation contributions disappear and the $L$-dependence disappears.

We remark that both effects, oscillatory (Eq. 14) and overlap (Eq. 15), depend explicitly on $Y$. Therefore, it is relevant to explore how the Effective Branching Ratio changes while $Y \equiv \Delta_{M_{N}} / \Gamma_{N}$ varies for a fixed value of $L$.

Figures 4 and 5 show the effective branching ratio as a function of $Y$ for different fixed maximal displaced vertex lengths (effective detector lengths) $L=300 \mathrm{~mm}$ and $L=$ $1000 \mathrm{~mm}$, respectively.

Figure 6 shows the $\mathrm{CP}$ asymmetry $\left(\mathrm{A}_{\mathrm{CP}}\right)$ as a function of the maximal displaced vertex length $L$ for three different values $Y$. Figure 7 shows the $\mathrm{CP}$ asymmetry as a function of $Y$ for three different values of length $L$.

On the other hand, some previous works (e.g. Ref. [100, 110]) have considered fixed values of $\gamma_{N}^{\prime \prime} \beta_{N}^{\prime \prime}$; in this scenario the CP asymmetry (Eq. 12) can be approximated as follows

$$
A_{\mathrm{CP}} \approx \sin \left(\theta_{L V}\right) \frac{\left[2-\exp \left(-L \Gamma_{N} /\left(\gamma_{N}^{\prime \prime} \beta_{N}^{\prime \prime}\right)\right)\left(1+\cos \left(\frac{2 \pi L}{L_{\mathrm{osc}}}\right)+\frac{1}{Y} \sin \left(\frac{2 \pi L}{L_{\mathrm{osc}}}\right)\right)\right] \frac{Y}{Y^{2}+1}}{\left[1+\frac{2}{Y^{2}+1} \cos \theta_{L V}\right]-\exp \left(-L \Gamma_{N} /\left(\gamma_{N}^{\prime \prime} \beta_{N}^{\prime \prime}\right)\right)\left[1+\frac{\cos \left(\theta_{L V}\right)}{Y^{2}+1}\left(1+\cos \left(\frac{2 \pi L}{L_{\mathrm{osc}}}\right)-Y \sin \left(\frac{2 \pi L}{L_{\mathrm{osc}}}\right)\right)\right]} .
$$

(b) or we can disregard in Eq. (13) the oscillatory terms and include only the overlap (resonant) terms

$$
\begin{aligned}
\Gamma_{\text {eff }}^{\text {overlap }} & \left(B^{ \pm} \rightarrow D^{0} \ell_{1}^{ \pm} \ell_{2}^{ \pm} \pi^{\mp}\right) \\
= & 2\left|B_{\ell_{1} N}\right|^{2}\left|B_{\ell_{2} N}\right|^{2} \frac{\bar{\Gamma}\left(N \rightarrow \ell_{2} \pi\right)}{\Gamma_{N}} \int d q^{2} \int d \Omega_{\hat{q}^{\prime}} \\
& \times \int d \Omega_{\hat{p}_{1}} \frac{d \bar{\Gamma}\left(B \rightarrow D \ell_{1} N\right)}{d q^{2} d \Omega_{\hat{q}^{\prime}} d \Omega_{\hat{p}_{1}}} \\
& \times\left[\left\{1-\exp \left(-L \Gamma_{N} / \sqrt{\left(E_{N}^{\prime \prime}\left(q^{2} ; \hat{q}^{\prime}, \hat{p}_{1}\right) / M_{N}\right)^{2}-1}\right)\right\}\right. \\
& \left.\times\left[1+\delta(Y) \cos \left(\theta_{L V}\right) \mp \frac{\eta(Y)}{Y} \sin \left(\theta_{L V}\right)\right]\right] .
\end{aligned}
$$

Figure 3 show a comparison between Eqs. (13), (14) and (15), as a function of the maximal displaced vertex length (effective detector length) $L$. We recall that the effective branching ratio is $\mathrm{Br}_{\text {eff }}=\Gamma_{\text {eff }} / \Gamma_{B}$, where $\Gamma_{B}=4.017 \times$ $10^{-13} \mathrm{GeV}$.

We can deduce from these figures that the oscillation contributions are usually larger in magnitude than the overlap (resonant) contributions, and that this trend gets stronger when $Y$ increases.

On the other hand, we notice that Fig. 3 show very small values of $\mathrm{Br}_{\text {eff }}$ when the detector length $L \approx 0$, this is consequent with the fact that at short distances only few neutrinos have decayed. On the contrary, for large $L$ all neutrinos have decayed, therefore the $\mathrm{Br}_{\text {eff }}$ becomes constant. In the expression Eq. (13) this situation is reflected when $L$ is so large
Figure 8 shows the $\mathrm{CP}$ asymmetry in Eq. 16 as a function of the maximal displaced vertex length $L$ (top) and $Y$ parameter (bottom)

\section{Discussion of the results and summary}

In this work we have studied the CP-violating effects in the rare $B$ meson decays mediated by two on-shell HNs. Unlike previous works, our calculations include both overlap (resonant) and oscillating effects. The variation of the values of the parameter $Y \equiv \Delta M_{N} / \Gamma_{N}$ shows that there exists a massdifference regime in which the CP-violating effects can be noticeable. Our formulas are approximations which are good if $Y$ is not too small $(Y \gtrsim 5)$, because we do not know (and do not include) the terms which are simultaneously overlap and oscillation effects. On the other hand, if $Y<1$, i.e., the mass difference $\Delta M_{N}$ is smaller than the decay width $\Gamma_{N}$, the CP-violating effects are expected to be highly suppressed and $\mathrm{A}_{\mathrm{CP}} \rightarrow 0$ as $Y \rightarrow 0$. We set the maximum value of the displaced vertex length (effective detector length) $L$ to $L=1000 \mathrm{~mm}$ in order to obtain a realistic prediction of the number of events that can take place at Belle II experiment.

While Fig. 3 show that in both cases $Y=5,10$ the oscillatory effects have a bigger contribution to the total effec-

\footnotetext{
$\overline{{ }^{6} \text { Here we will }}$ consider $\gamma_{N}^{\prime \prime} \beta_{N}^{\prime \prime}=0.664613$ which is the naive average value in the lab frame (when the weight function is constant).
} 

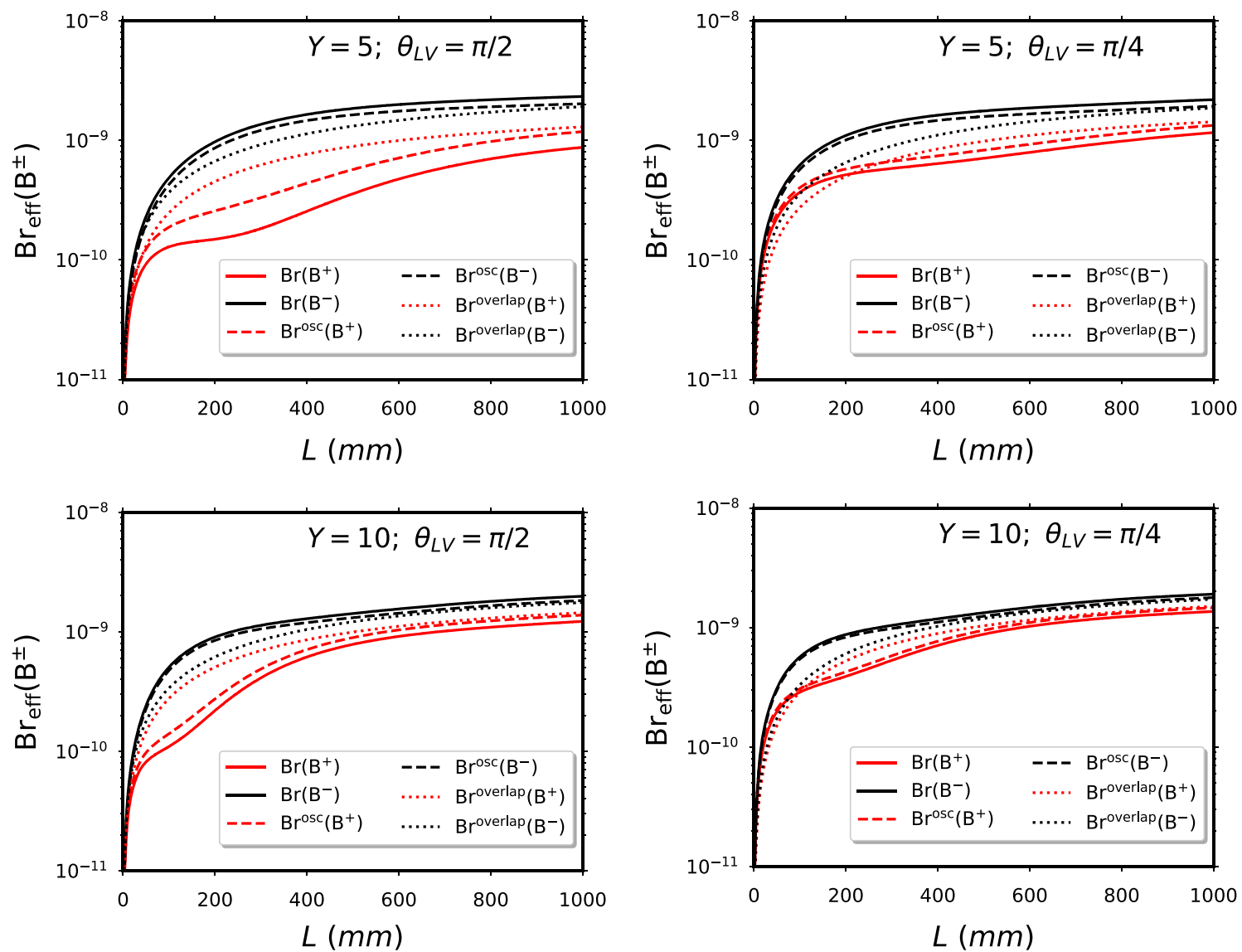

Fig. 3 Different contributions to the total effective branching ratio as a function of the maximal displaced vertex length $(L)$ for $M_{N}=2.0$ $\mathrm{GeV},\left|B_{\mu N}\right|^{2}=\left|B_{\tau N}\right|^{2}=10^{-5}$ and different values of $Y$ and $\theta_{L V}$.

Full lines stand for the case of $\Gamma_{\text {eff }}\left(B^{ \pm}\right)$(Eq. 13), dashed lines stand for $\Gamma_{\mathrm{eff}}^{\mathrm{osc}}\left(B^{ \pm}\right)$(Eq. 14) and dotted ones for $\Gamma_{\mathrm{eff}}^{\text {overlap }}\left(B^{ \pm}\right)$(Eq. 15). The curve for $B^{-}$are higher than those for $B^{+}$
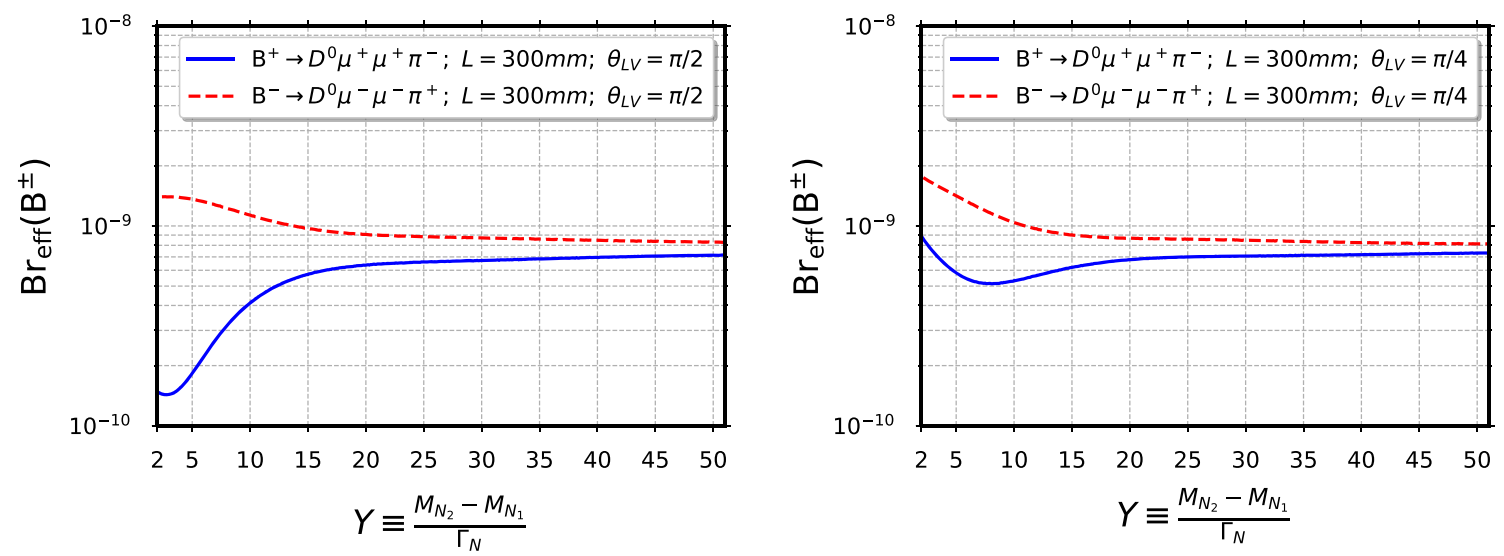

Fig. 4 Effective branching ratio as a function of $Y$ for $M_{N}=2.0 \mathrm{GeV}, L=300 \mathrm{~mm}$ and $\left|B_{\mu N}\right|^{2}=\left|B_{\tau N}\right|^{2}=10^{-5}$. Left Panel: $\theta_{L V}=\pi / 2$. Right panel: $\theta_{L V}=\pi / 4$ 

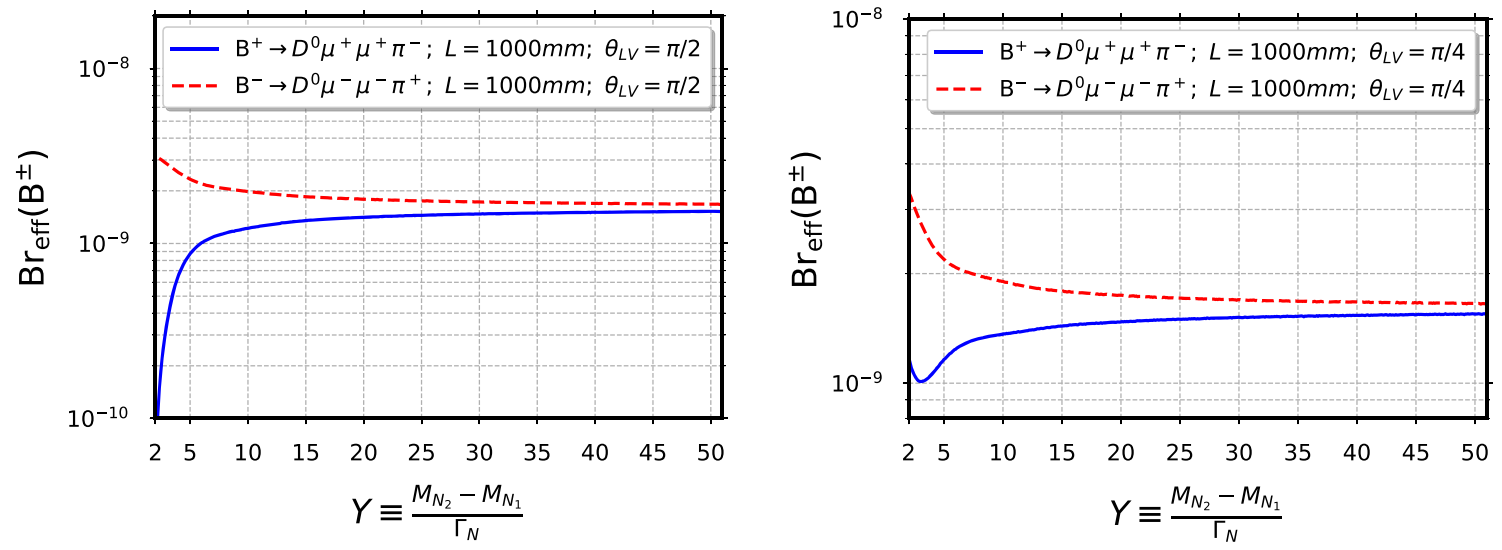

Fig. 5 Effective branching ratio as a function of $Y$ for $M_{N}=2.0 \mathrm{GeV}, L=1000 \mathrm{~mm}$ and $\left|B_{\mu N}\right|^{2}=\left|B_{\tau N}\right|^{2}=10^{-5}$. Left Panel: $\theta_{L V}=\pi / 2$. Right panel: $\theta_{L V}=\pi / 4$
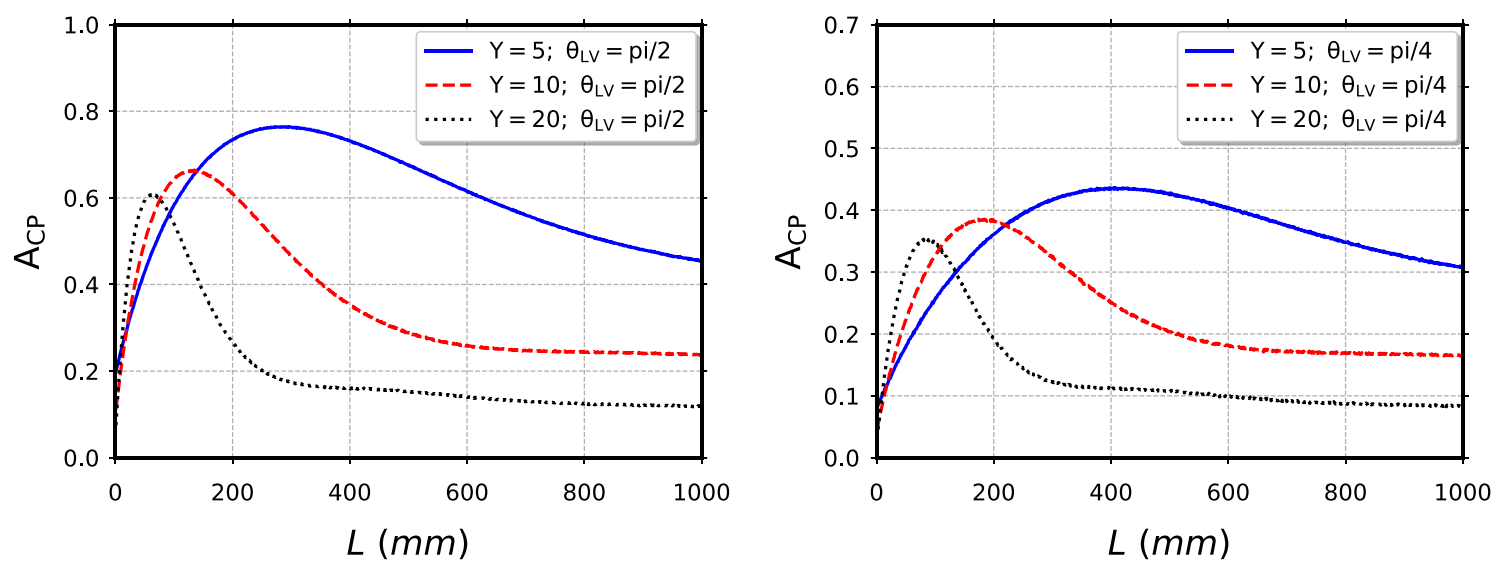

Fig. $6 \mathrm{CP}$ asymmetry as a function of the maximal displaced vertex length $(L)$ for $M_{N}=2.0 \mathrm{GeV}$ and $\left|B_{\mu N}\right|^{2}=\left|B_{\tau N}\right|^{2}=10^{-5}$. Left Panel: $\theta_{L V}=\pi / 2$. Right panel: $\theta_{L V}=\pi / 4$
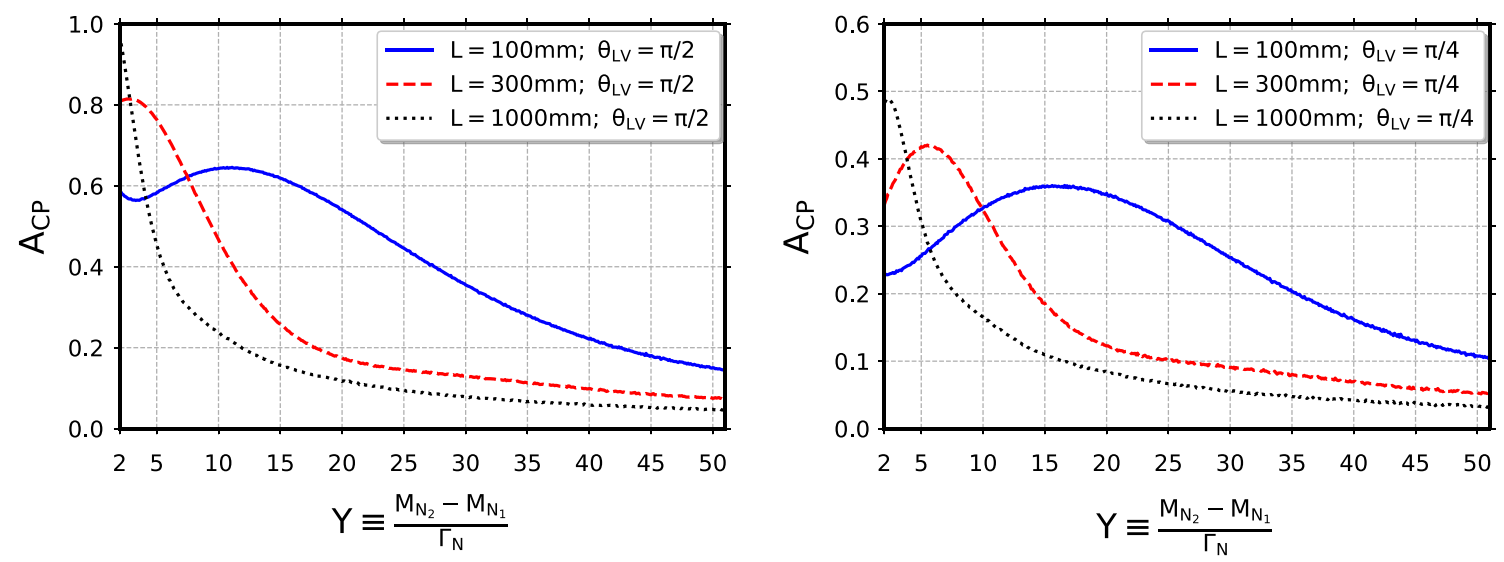

Fig. $7 \mathrm{CP}$ asymmetry as a function of $Y$ for $M_{N}=2.0 \mathrm{GeV}$ and $\left|B_{\mu N}\right|^{2}=\left|B_{\tau N}\right|^{2}=10^{-5}$. Left Panel: $\theta_{L V}=\pi / 2$. Right panel: $\theta_{L V}=\pi / 4$ 

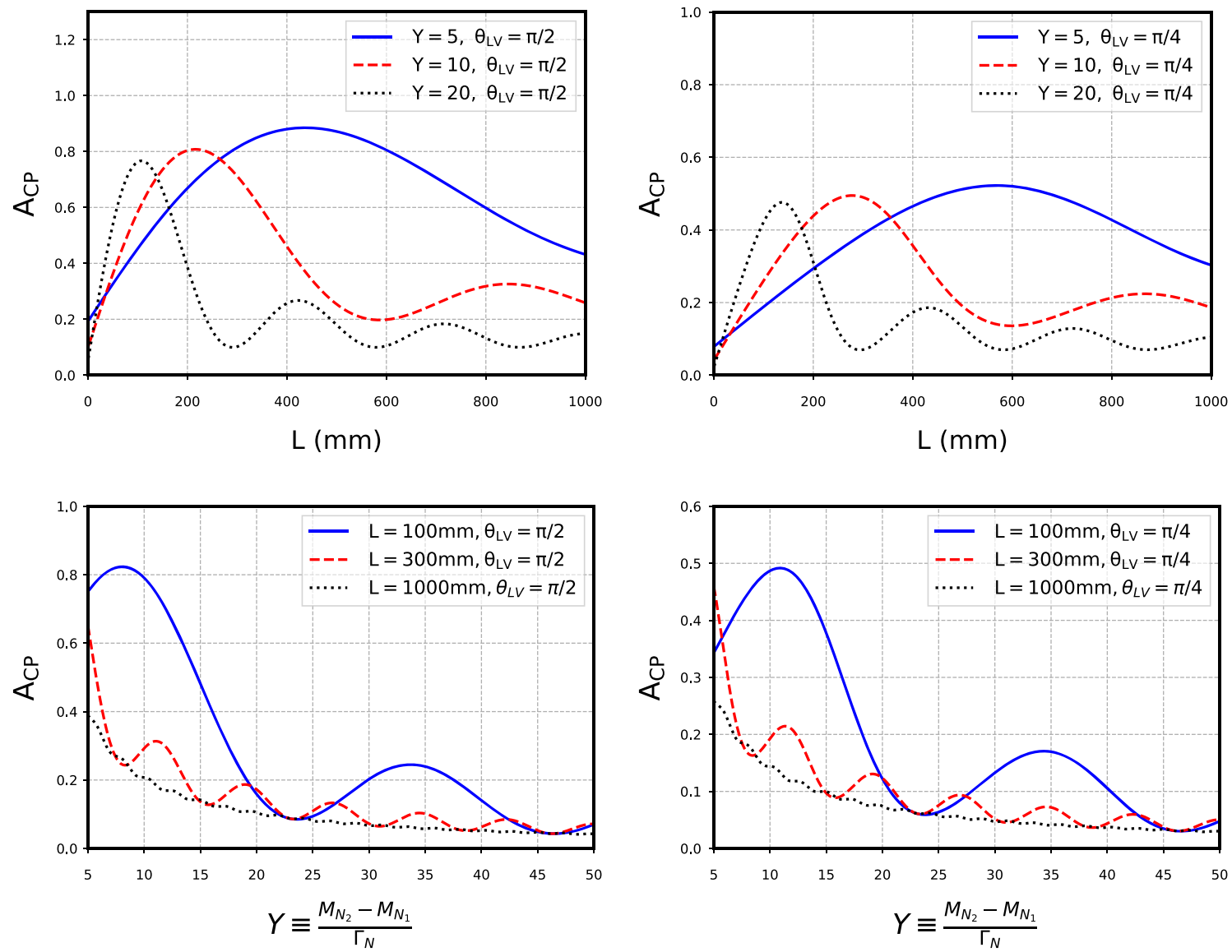

Fig. $8 \mathrm{CP}$ asymmetry for a fixed value of $\gamma_{N}^{\prime \prime} \beta_{N}^{\prime \prime}(=664613)$ (Eq. 16) for $M_{N}=2.0 \mathrm{GeV}$ and $\left|B_{\mu N}\right|^{2}=\left|B_{\tau N}\right|^{2}=10^{-5}$. Top panels show A as a function of $L$, bottom panels as a function of $Y$. Left panels: $\theta_{L V}=\pi / 2$. Right panels: $\theta_{L V}=\pi / 4$

tive branching ratio (full lines), we can see that both effects (oscillatory and overlap) contributions are of the same order of magnitude. In addition, Fig. 3 (top panel) we can see that the biggest difference from $B^{+}$and $B^{-}$effective branching ratios occurs between the 200 and $400 \mathrm{~mm}$. Furthermore, the channel difference changes with the CP violating phase $\theta_{L V}$, where the biggest $\mathrm{CP}$ violation appears at $\pi / 2$ and the smallest occurs at $\pi / 4$. For values of $\theta_{L V}=0, \pi$, there will be no difference between the channels. If the parameter $Y$ increases from 5 to 10 (Fig. 3, bottom panel) one can notice that now the biggest $\mathrm{CP}$ violation moves to the left and occurs between 50 and $200 \mathrm{~mm}$, while the maximum occurs at $\theta_{L V}=\pi / 2$.

The effect produced by the parameter $Y$ can be read from Figs. 4 and 5. Values of $Y>15$ shows little difference between the channels, this is well expected as for larger $Y$ the resonant and oscillating regimes will disappear when $\Delta M \propto Y \gg 1$. The maximum $\mathrm{CP}$ violation is strongly dependent on the length $L$, as seen from $L=300 \mathrm{~mm}$ in Fig. 4 and $L=1000 \mathrm{~mm}$ in Fig. 5 .
Figure 6 shows the asymmetry as a function of the length $L$. Although, the biggest value of the $\mathrm{CP}$ asymmetry appears for small values of the length $(L \sim 50-300 \mathrm{~mm})$, the branching ratios increase as $L \rightarrow 1000 \mathrm{~mm}$. Thus, biggest values of CP asymmetry are not enough to detect events. Therefore, the size of the branching ratios must also be taken into account in order to have a signal in the detector. Figure 7 shows the asymmetry as a function of $Y$. The biggest values of CP asymmetry appear for $Y=1-20$, and will disappear for $Y>50$. If we fix the value of $\gamma_{N}^{\prime \prime} \beta_{N}^{\prime \prime}$ (Fig. 8) we observe a clear oscillatory behaviour of $A_{C P}$. On the other hand, these effects are suppressed in Figs. 6 and $7\left(\gamma_{N}^{\prime \prime} \beta_{N}^{\prime \prime}\right.$ variable), due to the several integrations (average) performed in the evaluation of $A_{C P}$.

Moreover, Table 1 presents the expected number of events $\mathrm{N}_{\mathrm{e}}\left(\mathrm{B}^{ \pm}\right)=\eta \times \mathrm{N}_{\mathrm{B}} \times \mathrm{Br}_{\mathrm{eff}}\left(\mathrm{B}^{ \pm}\right)$, considering that the number of $B$ mesons expected at Belle II is $\mathrm{N}_{\mathrm{B}}=5 \times 10^{10}$. In addition, while the track reconstruction efficiency is greater than $90 \%$, we will consider it to be $\eta=30 \%$ in order to have a more conservative approach [116]. 
Table 1 Expected events at Belle II experiment. Here $\left|B_{\mu N}\right|^{2}=\left|B_{\tau N}\right|^{2}=10^{-5}$ and $M_{N}=2 \mathrm{GeV}$

\begin{tabular}{llllll}
\hline$L[\mathrm{~mm}]$ & $\mathrm{Y}$ & $\theta_{L V}$ & $\approx N_{e}\left(B^{-} \rightarrow D^{0} \mu^{-} \mu^{-} \pi^{+}\right)$ & $\approx N_{e}\left(B^{+} \rightarrow D^{0} \mu^{+} \mu^{+} \pi^{-}\right)$ & $\Delta N_{e} \equiv N_{e}\left(B^{-}\right)-N_{e}\left(B^{+}\right)$ \\
\hline 300 & 5 & $\pi / 2$ & 20 & 3 & 17 \\
300 & 10 & $\pi / 2$ & 17 & 6 & 11 \\
300 & 5 & $\pi / 4$ & 21 & 7 & 14 \\
300 & 10 & $\pi / 4$ & 16 & 13 & 8 \\
1000 & 5 & $\pi / 2$ & 35 & 18 & 22 \\
1000 & 10 & $\pi / 2$ & 30 & 17 & 12 \\
1000 & 5 & $\pi / 4$ & 33 & 20 & 9 \\
1000 & 10 & $\pi / 4$ & 29 &
\end{tabular}

In summary, in this work we studied the B-mesons decays $B^{ \pm} \rightarrow D^{0} \mu_{1}^{ \pm} \mu_{2}^{ \pm} \pi^{\mp}$ at Belle II, considering a $1000 \mathrm{~mm}$ effective detector length. We focused in a scenario with two almost-degenerate heavy neutrinos with masses around $M_{N} \sim 2 \mathrm{GeV}$. The effective branching ratios were calculated by considering that the heavy neutrino total decay width is equal for both, as a consequence of the assumption that the heavy-light mixing coefficients satisfy $\left|B_{\ell N_{1}}\right|=$ $\left|B_{\ell N_{2}}\right|\left(\equiv\left|B_{\ell N}\right|^{2}\right)$ for $\ell=\mu, \tau$. Further, we considered $\left|B_{\mu N}\right|^{2} \sim\left|B_{\tau N}\right|^{2} \sim 10^{-5} \gg\left|B_{e N}\right|^{2}$. The calculations were performed in a scenario that contains both the overlap (resonant) and oscillating CP-violating sources. We observed that the biggest difference of detectable events occurs for $Y=5$ and $\theta=\pi / 2$ (Table 1 ).

We established that for certain presently allowed regime of values of $\left|B_{\mu N}\right|^{2}, Y\left(\equiv \Delta M_{N} / \Gamma_{N}\right)$ and $\theta_{L V}$, and with $M_{N} \approx 2 \mathrm{GeV}$, the aforementioned effects can be observed at Belle II.

Acknowledgements This work was supported in part by FONDECYT (Chile) Grants No. 1180344 (G.C.) and No. 3180032 (J.Z.S.). The work of C.S.K. was supported by the National Research Foundation of Korea (NRF) grant funded by Korea government of the Ministry of Education, Science and Technology (MEST) (No. 2018R1A4A1025334).

Data Availability Statement This manuscript has no associated data or the data will not be deposited. [Authors' comment: There is no data. All the information concerning the article can be extracted directly from the equations there.]

Open Access This article is licensed under a Creative Commons Attribution 4.0 International License, which permits use, sharing, adaptation, distribution and reproduction in any medium or format, as long as you give appropriate credit to the original author(s) and the source, provide a link to the Creative Commons licence, and indicate if changes were made. The images or other third party material in this article are included in the article's Creative Commons licence, unless indicated otherwise in a credit line to the material. If material is not included in the article's Creative Commons licence and your intended use is not permitted by statutory regulation or exceeds the permitted use, you will need to obtain permission directly from the copyright holder. To view a copy of this licence, visit http://creativecomm ons.org/licenses/by/4.0/.

Funded by SCOAP ${ }^{3}$.

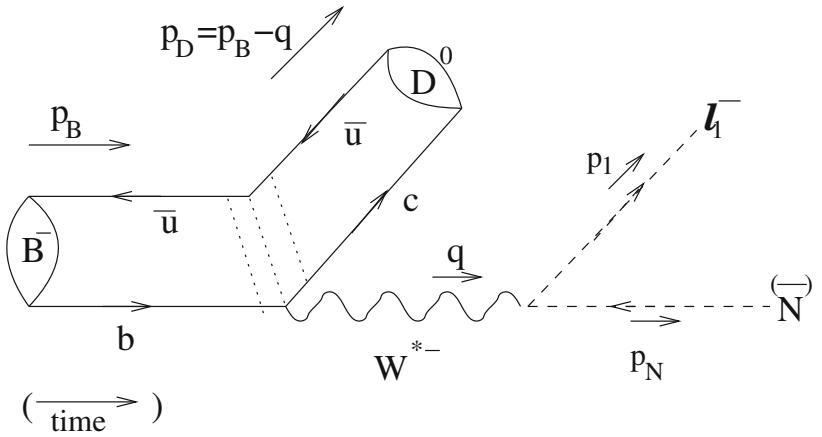

Fig. 9 Schematical representation of the decay $B^{-} \rightarrow D^{0} \ell_{1}^{-} \bar{N}[110]$

\section{Appendix A: Decay width $\Gamma\left(B \rightarrow D \ell_{1} N\right)$}

The differential decay width of the process $B \rightarrow D \ell_{1} N$ (see Fig. 9) was obtained in Ref. [111] and has the following form: ${ }^{7}$

$$
\begin{aligned}
& \frac{d \Gamma\left(B \rightarrow D \ell_{1} N\right)}{d q^{2} d \Omega_{\hat{q}^{\prime}} d \Omega_{\hat{p}_{1}}}=\left|B_{\ell_{1} N}\right|^{2} \frac{d \bar{\Gamma}\left(B \rightarrow D \ell_{1} N\right)}{d q^{2} d \Omega_{\hat{q}^{\prime}} d \Omega_{\hat{p}_{1}}} \\
& =\frac{\left|B_{\ell_{1} N}\right|^{2}\left|V_{c b}\right|^{2} G_{F}^{2}}{M_{B}(4 \pi)^{5}}|\widetilde{\mathcal{T}}|^{2} \lambda^{1 / 2}\left(1, \frac{M_{D}^{2}}{M_{B}^{2}}, \frac{q^{2}}{M_{B}^{2}}\right) \lambda^{1 / 2} \\
& \quad \times\left(1, \frac{M_{1}^{2}}{q^{2}}, \frac{M_{N}^{2}}{q^{2}}\right)
\end{aligned}
$$

We denote the $W^{*}$-rest frame (i.e., $\ell_{1} N$-rest frame) as $\Sigma$, and the $B$-rest frame as $\Sigma^{\prime}$ (where the corresponding momenta have a prime). In Eq. (A1), $q^{2}$ is the squared four-momentum of the $W^{*}$ boson, $\hat{q}^{\prime}$ is the unitary direction vector of $\mathbf{q}^{\prime}$ in the $B$-rest frame $\Sigma^{\prime}, \hat{p}_{1}$ is the unitary direction of $\mathbf{p}_{1}$ of $\ell_{1}$ in the $W^{*}$-rest $\left(\ell_{1} N\right.$-rest $)$ frame. In fact, $d \Omega_{\hat{p}_{1}}=d \phi_{1} d\left(\cos \theta_{1}\right)$

\footnotetext{
$\overline{7}$ In Ref. [111] there is a typo in Eq. (11) for this differential decay width, the expression given there must be multiplied by 4 . The correct formula was used in the calculations there, though, which reproduces the decay width $\Gamma\left(B \rightarrow D \ell_{1} N\right)$ calculated earlier in Ref. [110].
} 
where $\hat{z}=\hat{q}^{\prime}$. The expression $|\widetilde{\mathcal{T}}|^{2}$ stands for the squared decay amplitude and is given by

$$
\begin{aligned}
|\widetilde{\mathcal{T}}|^{2}= & \frac{1}{q^{2}} F_{1}\left(q^{2}\right)\left(F_{0}\left(q^{2}\right)-F_{1}\left(q^{2}\right)\right)\left(M_{B}^{2}-M_{D}^{2}\right) \\
& \times\left[M _ { 1 } ^ { 2 } \left(-4\left(\cos \theta_{1}\left|\mathbf{p}_{D}\right|\left|\mathbf{p}_{N}\right|+p_{D}^{0} p_{1}^{0}\right)\right.\right. \\
& \left.+2 M_{B}^{2}-2 M_{D}^{2}+2 M_{N}^{2}-q^{2}\right) \\
& +M_{N}^{2}\left(4 \left(\cos \theta_{1}\left|\mathbf{p}_{D}\right|\left|\mathbf{p}_{N}\right|\right.\right. \\
& \left.\left.\left.+p_{D}^{0} p_{1}^{0}\right)-M_{N}^{2}+q^{2}\right)-M_{1}^{4}\right] \\
& -\frac{1}{2} F_{1}\left(q^{2}\right)^{2}\left[M _ { 1 } ^ { 2 } \left(8\left(\cos \theta_{1}\left|\mathbf{p}_{D}\right|\left|\mathbf{p}_{N}\right|+p_{D}^{0} p_{1}^{0}\right)\right.\right. \\
& \left.-4 M_{B}^{2}-2 M_{N}^{2}+3 q^{2}\right) \\
& -8 M_{B}^{2}\left(\cos \theta_{1}\left|\mathbf{p}_{D}\right|\left|\mathbf{p}_{N}\right|+p_{D}^{0} p_{1}^{0}\right) \\
& +M_{D}^{2}\left(8\left(\cos \theta_{1}\left|\mathbf{p}_{D}\right|\left|\mathbf{p}_{N}\right|+p_{D}^{0} p_{1}^{0}\right)-4 M_{N}^{2}+4 q^{2}\right) \\
& -8 M_{N}^{2}\left(\cos \theta_{1}\left|\mathbf{p}_{D}\right|\left|\mathbf{p}_{N}\right|+p_{D}^{0} p_{1}^{0}\right) \\
& +8 q^{2}\left(\cos \theta_{1}\left|\mathbf{p}_{D}\right|\left|\mathbf{p}_{N}\right|+p_{D}^{0} p_{1}^{0}\right) \\
& +16\left(\cos \theta_{1}\left|\mathbf{p}_{D}\right|\left|\mathbf{p}_{N}\right|+p_{D}^{0} p_{1}^{0}\right)^{2} \\
& \left.+M_{1}^{4}+M_{N}^{4}-M_{N}^{2} q^{2}\right] \\
& +\frac{1}{2\left(q^{2}\right)^{2}}\left(F_{0}\left(q^{2}\right)-F_{1}\left(q^{2}\right)\right)^{2}\left(M_{B}^{2}-M_{D}^{2}\right)^{2}\left[-M_{1}^{4}\right. \\
& \left.+M_{1}^{2}\left(2 M_{N}^{2}+q^{2}\right)-M_{N}^{4}+M_{N}^{2} q^{2}\right]
\end{aligned}
$$

where

$$
\begin{aligned}
\left|\mathbf{p}_{N}\right| & =\left|\mathbf{p}_{1}\right|=\frac{1}{2} \sqrt{q^{2}} \lambda^{1 / 2}\left(1, \frac{M_{1}^{2}}{q^{2}}, \frac{M_{N}^{2}}{q^{2}}\right), \\
\left|\mathbf{p}_{D}\right| & =\frac{M_{B}^{2}}{2 \sqrt{q^{2}}} \lambda^{1 / 2}\left(1, \frac{M_{D}^{2}}{M_{B}^{2}}, \frac{q^{2}}{M_{B}^{2}}\right)=\frac{M_{B}\left|\mathbf{q}^{\prime}\right|}{\sqrt{q^{2}}}, \\
p_{1}^{0} & =\frac{1}{2 \sqrt{q^{2}}}\left(q^{2}-M_{N}^{2}+M_{1}^{2}\right), \\
p_{D}^{0} & =\frac{1}{2 \sqrt{q^{2}}}\left(M_{B}^{2}-M_{D}^{2}-q^{2}\right) .
\end{aligned}
$$

These momenta are all in the $W^{*}$-rest frame $(\Sigma) ; \theta_{1}$ is the angle between $\mathbf{p}_{1}$ and $\hat{z} \equiv \hat{q}^{\prime}$ (the direction of $W^{*}$ in the $B$-rest frame).

The expression (A2) is defined in terms of two form factors, $F_{1}$ and $F_{0}$. The form factor $F_{1}\left(q^{2}\right)$ is presented in [117] and is expressed in terms of $w\left(q^{2}\right)$ and $z(w)$

$$
\begin{aligned}
w\left(q^{2}\right) & =\frac{\left(M_{B}^{2}+M_{D}^{2}-q^{2}\right)}{2 M_{B} M_{D}}, \\
z(w) & =\frac{\sqrt{w+1}-\sqrt{2}}{\sqrt{w+1}+\sqrt{2}} .
\end{aligned}
$$

Therefore, from Ref. [117], $F_{1}\left(q^{2}\right)$ can be expressed as

$$
\begin{aligned}
F_{1}\left(q^{2}\right)= & F_{1}(w=1)\left(1-8 \rho^{2} z(w)+\left(51 \rho^{2}-10\right) z(w)^{2}\right. \\
& \left.-\left(252 \rho^{2}-84\right) z(w)^{3}\right) .
\end{aligned}
$$

In the last equation the free parameters $\rho^{2}$ and $F_{1}(w=1)$ have been determined by the Belle Collaboration [118]

$$
\begin{aligned}
\rho^{2} & =1.09 \pm 0.05, \\
\left|V_{c b}\right| F_{1}(w=1) & =(48.14 \pm 1.56) \times 10^{-3} .
\end{aligned}
$$

The form factor $F_{0}\left(q^{2}\right)$ is given as $[117]^{8}$

$$
\begin{aligned}
F_{0}\left(q^{2}\right)= & \frac{\left(M_{B}+M_{D}\right)}{2 \sqrt{M_{B} M_{D}}} \\
& \times\left[1-\frac{q^{2}}{\left(M_{B}+M_{D}\right)^{2}}\right] f_{0}\left(w\left(q^{2}\right)\right), \\
f_{0}(w) \approx & f_{0}(w=1)\left[1-\rho_{0}^{2}(w-1)\right. \\
& \left.+\left(0.72 \rho_{0}^{2}-0.09\right)(w-1)^{2}\right]
\end{aligned}
$$

where $f_{0}(w=1) \approx 1.02$ and $\rho_{0}^{2} \approx 1.102$.

The decay width for $B \rightarrow D \ell_{1} N$ decays is

$$
\begin{aligned}
& \Gamma\left(B \rightarrow D \ell_{1} N\right) \\
& =\left|B_{\ell_{1} N}\right|^{2} \int d q^{2} \int d \Omega_{\hat{q}^{\prime}} \int d \Omega_{\hat{p}_{1}} \frac{d \bar{\Gamma}\left(B \rightarrow D \ell_{1} N\right)}{d q^{2} d \Omega_{\hat{q}^{\prime}} d \Omega_{\hat{p}_{1}}},
\end{aligned}
$$

For the effective decay width, which takes into account only those decays in which the exchanged on-shell $N$ decays within the detector, we refer to Appendix C.

\section{Appendix B: Decay width for $N \rightarrow \ell^{ \pm} \pi^{\mp}$}

The decay width $\Gamma\left(N \rightarrow \ell^{ \pm} \pi^{\mp}\right)$ (see Fig. 10) is proportional to the heavy-light mixing factor $\left|U_{\ell_{2} N}\right|^{2}$

$\Gamma\left(N \rightarrow \ell^{ \pm} \pi^{\mp}\right)=\left|B_{\ell_{2} N}\right|^{2} \bar{\Gamma}\left(N \rightarrow \ell^{ \pm} \pi^{\mp}\right)$.

Here, the canonical decay width $\bar{\Gamma}$ is

$$
\begin{aligned}
\bar{\Gamma} & \left(N \rightarrow \ell^{ \pm} \pi^{\mp}\right) \\
= & \frac{1}{16 \pi}\left|V_{u d}\right|^{2} G_{F}^{2} f_{\pi}^{2} M_{N}^{3} \lambda^{1 / 2}\left(1, x_{\pi}, x_{\ell}\right)\left[1-x_{\pi}\right. \\
& \left.\quad-2 x_{\ell}-x_{\ell}\left(x_{\pi}-x_{\ell}\right)\right],
\end{aligned}
$$

where $f_{\pi}(\approx 0.1304 \mathrm{GeV})$ is the decay constant of pion, and the other factors are

$x_{\pi}=\frac{M_{\pi}^{2}}{M_{N}^{2}}, \quad x_{\ell}=\frac{M_{\ell}^{2}}{M_{N}^{2}}$.

\footnotetext{
$\overline{8}$ In Ref. [110],$f_{0}(w)$ was transcribed there in Eq. (11b) with a typo $\left[+\rho_{0}^{2}(w-1)\right.$ instead of $\left.-\rho_{0}^{2}(w-1)\right]$, but the correct expression (A6b) was used in the calculations there.
} 


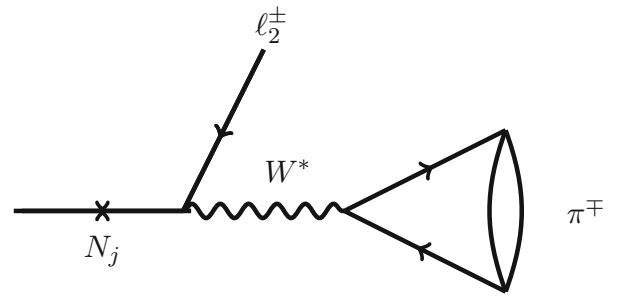

Fig. 10 Feynmann diagram for the decay process $N \rightarrow \ell^{ \pm} \pi^{\mp}$

These results can be combined with the result (A7) to obtain the decay width for the decay $B^{ \pm} \rightarrow D^{0} \ell_{1}^{ \pm} N \rightarrow$ $\ell_{1}^{ \pm} \ell_{2}^{ \pm} \pi^{\mp}$

$$
\begin{aligned}
\Gamma & \left(B^{ \pm} \rightarrow D^{0} \ell_{1}^{ \pm} N \rightarrow \ell_{1}^{ \pm} \ell_{2}^{ \pm} \pi^{\mp}\right) \\
= & \Gamma\left(B^{ \pm} \rightarrow D^{0} \ell_{1}^{ \pm} N\right) \frac{\Gamma\left(N \rightarrow \ell_{2}^{ \pm} \pi^{\mp}\right)}{\Gamma_{N}}, \\
= & \left|B_{\ell_{1} N}\right|^{2}\left|B_{\ell_{2} N}\right|^{2} \frac{\bar{\Gamma}\left(N \rightarrow \ell_{2} \pi\right)}{\Gamma_{N}} \int d q^{2} \int d \Omega_{\hat{q}^{\prime}} \\
& \times \int d \Omega_{\hat{p}_{1}} \frac{d \bar{\Gamma}\left(B \rightarrow D \ell_{1} N\right)}{d q^{2} d \Omega_{\hat{q}^{\prime}} d \Omega_{\hat{p}_{1}}},
\end{aligned}
$$

where the expressions (A1) and (A7) are used for the first factor, and (B1) and (B2) for the second factor of the integrand. For $\Gamma_{N}$ we refer to Eq. (10).

\section{Appendix C: Lorentz factors of on-shell $N$ in laboratory frame}

In this Appendix we follow the presentation given in Ref. [111]. The expression (B4) refers to the decay width for all the decays of the type $B^{ \pm} \rightarrow D^{0} \ell_{1}^{ \pm} N \rightarrow \ell_{1}^{ \pm} \ell_{2}^{ \pm} \pi^{\mp}$, including those where the on-shell $N$ decays outside the detector. However, if we realistically consider that only those decays are detected in which the on-shell $N$ decays within the detector (of length $L$ ), we need to multiply the integrand in Eq. (B4) with the probability $P_{N}$ of decaying of the produced on-shell $N$ within the length $L$.

$P_{N}=1-\exp \left[-\frac{L}{\tau_{N} \gamma_{N}^{\prime \prime} \beta_{N}^{\prime \prime}}\right]=1-\exp \left[-\frac{L \Gamma_{N}}{\gamma_{N}^{\prime \prime} \beta_{N}^{\prime \prime}}\right]$,

where $\tau_{N}=1 / \Gamma_{N}$ is the lifetime of $N$ in its rest frame. The velocity $\beta_{N}^{\prime \prime}$ and the Lorentz factor $\gamma_{N}^{\prime \prime}=1 / \sqrt{1-\left(\beta_{N}^{\prime \prime}\right)^{2}}$ are those of the $N$ neutrino in the lab frame $\Sigma^{\prime \prime}$.

\footnotetext{
${ }^{9}$ We use the same conventions as in Appendix A: the $W^{*}$-rest frame $\left(\ell_{1} N\right.$-rest frame) is $\Sigma$, and the $B$-rest frame is $\Sigma^{\prime}$. The lab frame is denoted as $\Sigma^{\prime \prime}$ (and the corresponding momenta have double prime). Note, however, that the distance between the two vertices of the on-shell $N$ in the lab frame is denoted for simplicity as $L$ (and not: $L^{\prime \prime}$ ).
}

At Belle II, the kinetic energy of the produced $\Upsilon(4 S)$ is $0.421 \mathrm{GeV}$, and this implies that its Lorentz factor in the lab frame $\Sigma^{\prime \prime}$ is $\gamma_{\Upsilon}^{\prime \prime}=1.0398$ and $\beta_{\Upsilon}^{\prime \prime}=0.274$. When $\Upsilon(4 S)$ produces a pair of $B$ mesons, the kinetic energy of $B$ mesons in the $\Upsilon$-rest frame is $0.010 \mathrm{GeV}$, which is negligible. Thus the velocity of the $B$ mesons in the lab frame $\Sigma^{\prime \prime}$ is equal to the velocity of $\Upsilon(4 S)$

$\beta_{B}^{\prime \prime}=0.274, \quad \gamma_{B}^{\prime \prime}=1.0398$.

Then, the factor $\gamma_{N}^{\prime \prime} \beta_{N}^{\prime \prime}$ appearing in the probability (C1) can be calculated by calculating the energy $E_{N}^{\prime \prime}$ of the $N$ neutrino in the lab frame (see below)

$\gamma_{N}^{\prime \prime} \beta_{N}^{\prime \prime}=\sqrt{\left(E_{N}^{\prime \prime}\left(q^{2} ; \hat{q}^{\prime}, \hat{p}_{1}\right) / M_{N}\right)^{2}-1}$,

and this leads to the effective decay width for the considered process

$$
\begin{aligned}
\Gamma_{\text {eff }} & \left(B \rightarrow D \ell_{1} N \rightarrow D \ell_{1} \ell_{2} \pi\right) \\
= & \left|B_{\ell_{1} N}\right|^{2}\left|B_{\ell_{2} N}\right|^{2} \frac{\bar{\Gamma}\left(N \rightarrow \ell_{2} \pi\right)}{\Gamma_{N}} \int d q^{2} \int d \Omega_{\hat{q}^{\prime}} \\
& \times \int d \Omega_{\hat{p}_{1}} \frac{d \bar{\Gamma}\left(B \rightarrow D \ell_{1} N\right)}{d q^{2} d \Omega_{\hat{q}^{\prime}} d \Omega_{\hat{p}_{1}}} \\
& \times\left\{1-\exp \left[-\frac{L \Gamma_{N}}{\sqrt{\left(E_{N}^{\prime \prime}\left(q^{2} ; \hat{q}^{\prime}, \hat{p}_{1}\right) / M_{N}\right)^{2}-1}}\right]\right\},
\end{aligned}
$$

which is as the expression (B4) but with inclusion of the $N$ decay probability within the effective detector length $L .^{10}$ The energy $E_{N}^{\prime \prime}$ of the produced heavy neutrino $N$ in the lab frame and is given by (cf. App. B of Ref. [111])

$$
\begin{aligned}
E_{N}^{\prime \prime} & \left(q^{2} ; \theta_{q} ; \theta_{1}, \phi_{1}\right) \\
= & \gamma_{B}\left(\gamma_{W}\left(q^{2}\right)\left(E_{N}\left(q^{2}\right)-\beta_{W}\left(q^{2}\right)\left|\mathbf{p}_{N}\left(q^{2}\right)\right| \cos \theta_{1}\right)\right. \\
& +\beta_{B}\left[\gamma _ { W } ( q ^ { 2 } ) \left(-\left|\mathbf{p}_{N}\left(q^{2}\right)\right| \cos \theta_{1}\right.\right. \\
& \left.+\beta_{W}\left(q^{2}\right) E_{N}\left(q^{2}\right)\right) \cos \theta_{q} \\
& \left.\left.-\left|\mathbf{p}_{N}\left(q^{2}\right)\right| \sin \theta_{1} \cos \phi_{1} \sin \theta_{q}\right]\right)
\end{aligned}
$$

The factors, as a function of the squared invariant mass of $W^{*}, q^{2}$ (see Fig. 9), are

$$
E_{N}=\frac{1}{2 \sqrt{q^{2}}}\left(q^{2}+M_{N}^{2}-M_{1}^{2}\right)
$$

10 The effective detector length here is considered to be independent of the position of the $N$-production vertex and independent of the direction in which the produced $N$ travels. 


$$
\begin{aligned}
\left|\mathbf{p}_{N}\right| & =\left|\mathbf{p}_{1}\right|=\frac{1}{2} \sqrt{q^{2}} \lambda^{1 / 2}\left(1, \frac{M_{1}^{2}}{q^{2}}, \frac{M_{N}^{2}}{q^{2}}\right), \\
\gamma_{W}\left(q^{2}\right) & =\left(1+\frac{\left|\mathbf{q}^{\prime}\right|^{2}}{q^{2}}\right)^{1 / 2}, \quad \beta_{W}\left(q^{2}\right)=\left(\frac{q^{2}}{\left|\mathbf{q}^{\prime}\right|^{2}}+1\right)^{-1 / 2},
\end{aligned}
$$

and

$$
\left|\mathbf{q}^{\prime}\right|=\frac{1}{2} M_{B} \lambda^{1 / 2}\left(1, \frac{M_{D^{*}}^{2}}{M_{B}^{2}}, \frac{q^{2}}{M_{B}^{2}}\right),
$$

see also Eq. (A3). In Eq. (C5) the angles $\theta_{q}, \theta_{1}$ and $\phi_{1}$ are in the following ranges:

$0 \leq \theta_{q} \leq \pi$

$0 \leq \theta_{1} \leq \pi$,

$0 \leq \phi_{1}<2 \pi$.

For a more detailed explanation of the aforemention expressions we refer to Ref. [111].

\section{Appendix D: Effective width of the LNV B decay channel with overlap and oscillation effects}

Here we will explain how the expression (6) is obtained. We work in the case when the Lorentz factors in the lab frame $\beta_{N}^{\prime \prime}$ and $\gamma_{N}^{\prime \prime} \equiv 1 / \sqrt{1-\left(\beta_{N}^{\prime \prime}\right) 2}$ are considered to be fixed. In addition, we use the assumption made throughtout this work that the heavy-light mixing elements satisfy $\left|B_{\ell N_{1}}\right|=\left|B_{\ell N_{2}}\right|$ ( $\equiv\left|B_{\ell N}\right|$ ), where $\ell=\mu, e, \tau$. When no oscillation is assumed [i.e., only the overlap (resonant) effects included], the effective decay width for the considered LNV decay channnel is [98] [cf. also [103] Eq. (13) there]

$$
\begin{aligned}
\Gamma_{\text {eff }} & \left(B^{ \pm} \rightarrow D^{0} \ell_{1}^{ \pm} \ell_{2}^{ \pm} \pi^{\mp}\right)_{\text {res }} \\
= & \Gamma\left(B^{ \pm} \rightarrow D^{0} \ell_{1}^{ \pm} N\right) \frac{\Gamma\left(N \rightarrow \ell_{2}^{ \pm} \pi^{\mp}\right)}{\Gamma_{N}} 2\left|B_{\ell_{1} N}\right|^{2}\left|B_{\ell_{2} N}\right|^{2} \\
& \times\left[1-\exp \left(-L \Gamma_{N} /\left(\gamma_{N}^{\prime \prime} \beta_{N}^{\prime \prime}\right)\right)\right]\left[1+\delta(Y) \cos \left(\theta_{L V}\right)\right. \\
& \left.\mp \frac{\eta(Y)}{Y} \sin \left(\theta_{L V}\right)\right] .
\end{aligned}
$$

We recall that $L$ here is the length of flight of the on-shell $N_{j}$ in the detector before it decays (within the detector), and the parameter $Y$ and the $N_{1}-N_{2}$ overlap functions $\delta(Y)$ and $\eta(Y)$ are given in Eqs. (7) and (9). The differential decay rate $d \Gamma_{\text {eff }} / d L$ for this decay width is then

$$
\begin{aligned}
\left(\frac{d \Gamma_{\text {eff }}}{d L}\right)_{\text {res }}= & \frac{1}{\gamma_{N}^{\prime \prime} \beta_{N}^{\prime \prime}} \exp \left(-\frac{L \Gamma_{N}}{\gamma_{N}^{\prime \prime} \beta_{N}^{\prime \prime}}\right) \Gamma\left(B^{ \pm} \rightarrow D^{0} \ell_{1}^{ \pm} N\right) \Gamma(N \\
& \left.\rightarrow \ell_{2}^{ \pm} \pi^{\mp}\right) 2\left|B_{\ell_{1} N}\right|^{2}\left|B_{\ell_{2} N}\right|^{2} \\
& \times\left[1+\delta(Y) \cos \left(\theta_{L V}\right) \mp \frac{\eta(Y)}{Y} \sin \left(\theta_{L V}\right)\right]
\end{aligned}
$$

On the other hand, when $Y \gg 1$ and thus the overlap contributions $\sim \delta(Y)$ and $\sim \eta(Y) / Y$ can be neglected, we obtained in Ref. [103] the corresponding differential decay width with $N_{1}-N_{2}$ oscillation effects included ${ }^{11}$

$$
\begin{aligned}
\left(\frac{d \Gamma_{\mathrm{eff}}}{d L}\right)_{\mathrm{osc}}= & \frac{1}{\gamma_{N}^{\prime \prime} \beta_{N}^{\prime \prime}} \exp \left(-\frac{L \Gamma_{N}}{\gamma_{N}^{\prime \prime} \beta_{N}^{\prime \prime}}\right) \Gamma\left(B^{ \pm}\right. \\
& \left.\rightarrow D^{0} \ell_{1}^{ \pm} N\right) \Gamma\left(N \rightarrow \ell_{2}^{ \pm} \pi^{\mp}\right) 2\left|B_{\ell_{1} N}\right|^{2}\left|B_{\ell_{2} N}\right|^{2} \\
& \times\left[1+\cos \left(2 \pi \frac{L}{L_{\mathrm{osc}}} \pm \theta_{L V}\right)\right]
\end{aligned}
$$

where $L_{\mathrm{osc}}$ is the $\mathrm{HN}$ oscillation length

$L_{\mathrm{osc}}=\frac{2 \pi \gamma_{N}^{\prime \prime} \beta_{N}^{\prime \prime}}{\Delta M_{N}} \Rightarrow 2 \pi \frac{L}{L_{\mathrm{osc}}}=Y \frac{\Gamma_{N}}{\gamma_{N}^{\prime \prime} \beta_{N}^{\prime \prime}} L$

If we now combine the overlap (resonant) contributions contained in the expression (D2) with the oscillation contributions contained in the expression (D3), we obtain

$$
\begin{aligned}
& \left(\frac{d \Gamma_{\text {eff }}}{d L}\right)=\frac{1}{\gamma_{N}^{\prime \prime} \beta_{N}^{\prime \prime}} \exp \left(-\frac{L \Gamma_{N}}{\gamma_{N}^{\prime \prime} \beta_{N}^{\prime \prime}}\right) \Gamma\left(B^{ \pm}\right. \\
& \left.\rightarrow D^{0} \ell_{1}^{ \pm} N\right) \Gamma\left(N \rightarrow \ell_{2}^{ \pm} \pi^{\mp}\right) 2\left|B_{\ell_{1} N}\right|^{2}\left|B_{\ell_{2} N}\right|^{2} \\
& \quad \times\left\{1+\left[\delta(Y) \cos \left(\theta_{L V}\right) \mp \frac{\eta(Y)}{Y} \sin \left(\theta_{L V}\right)\right]\right. \\
& \left.\quad+\cos \left(2 \pi \frac{L}{L_{\mathrm{osc}}} \pm \theta_{L V}\right)\right\}
\end{aligned}
$$

The expression (D3) was obtained in Ref. [103] from the expression (D2) under the assumption that the overlap contributions $(\sim \delta(Y), \eta(Y) / Y)$ there were negligible, i.e., that $Y \gg 1$. Combination of these two expressions into the expression (D5) thus involves an approximation of neglecting oscillation terms which involve overlap effects, i.e., terms of the type $\sim(\eta(Y) / Y) \cos \left(2 \pi L / L_{\text {osc }} \pm \theta_{L V}\right)$ or similar (we do not know these terms). ${ }^{12}$ This approximation is also reflected in the fact that the expression (D5) is negative for some flight lengths $L$, which should not happen. However, if $Y$ is significantly larger than one (say, $Y \gtrsim 5$ ), these negative contributions are small in absolute value and appear only in very short intervals of $L$, and consequently the expression

\footnotetext{
11 In [103] we wrote this expression in the approximation of small $N_{j}$-decay probability $P_{N}(L) \equiv\left[1-\exp \left(-L \Gamma_{N} /\left(\gamma_{N}^{\prime \prime} \beta_{N}^{\prime \prime}\right)\right)\right]$, namely $P_{N}(L) \approx L \Gamma_{N} /\left(\gamma_{N}^{\prime \prime} \beta_{N}^{\prime \prime}\right)$. In Refs. [104,105] and here we wrote this expression without this approximation, which gives us an additional factor $\exp \left(-L \Gamma_{N} /\left(\gamma_{N}^{\prime \prime} \beta_{N}^{\prime \prime}\right)\right)$ in $d P_{N}(L) / d L$ and in $\left(d \Gamma_{\text {eff }} / d L\right)_{\text {osc }}$.

12 One may be worried that this hierarchical view may not be adequate, because Eq. (D5) for $d \Gamma_{\text {eff }} / d L$ may suggest that the overlap effects (at $Y \gg 1)$ are smaller than the oscillation effects. Nonetheless, the two types of effects are mutually comparable in the integrated width $\Gamma_{\text {eff }}$ (cf. also the last paragraph in this Appendix).
} 
(D5) can be regarded as a reasonably good approximation containing simultaneously both the overlap (resonant) and oscillation contributions, especially when it is integrated over $L$.

Integration of the partial decay width (D5) from $L=0$ to $L$ then gives us the expression (6) in Sect. 2 [expresssion (13) in Sect. (3)], where we can clearly see that the overlap contribution and the oscillation contribution to $\Gamma_{\text {eff }}(B \rightarrow$ $\left.D \ell_{1} \ell_{2} \pi\right)$ are mutually comparable, in view of the relations (9).

\section{References}

1. Y. Fukuda et al. (Super-Kamiokande), Phys. Rev. Lett. 81, 1562 (1998). arXiv:hep-ex/9807003. https://doi.org/10.1103/ PhysRevLett.81.1562

2. K. Eguchi et al. (KamLAND), Phys. Rev. Lett. 90, 021802 (2003). arXiv:hep-ex/0212021 [hep-ex]. https://doi.org/10.1103/ PhysRevLett.90.021802

3. B. Pontecorvo, Sov. Phys. JETP 7, 172 (1958)

4. Q. Ahmad et al. (SNO), Phys. Rev. Lett. 89, 011301 (2002). arXiv:nucl-ex/0204008. https://doi.org/10.1103/PhysRevLett. 89.011301

5. P. Lipari, Phys. Rev. D 64, 033002 (2001). https://doi.org/10. 1103/PhysRevD.64.033002. arXiv:hep-ph/0102046

6. Z. Rahman, A. Dasgupta, R. Adhikari (2012). arXiv:1210.2603 [hep-ph]

7. A. Dasgupta, Z. Rahman, R. Adhikari (2012). arXiv:1210.4801 [hep-ph]

8. M. Gell-Mann, P. Ramond, R. Slansky, Conf. Proc. C 790927, 315 (1979). arXiv:1306.4669 [hep-th]

9. O. Sawada, A. Sugamoto, eds., Proceedings: Workshop on the Unified Theories and the Baryon Number in the Universe: Tsukuba, Japan, February 13-14, 1979 (Natl.Lab.High Energy Phys., Tsukuba, Japan, 1979)

10. R. N. Mohapatra, G. Senjanović, Phys. Rev. Lett. 44, 912 (1980).https://doi.org/10.1103/PhysRevLett.44.912

11. D. Wyler, L. Wolfenstein, Nucl. Phys. B 218, 205 (1983). https:// doi.org/10.1016/0550-3213(83)90482-0

12. E. Witten, Nucl. Phys. B 258, 75 (1985). https://doi.org/10.1016/ 0550-3213(85)90603-0

13. R. Mohapatra J. Valle, Phys. Rev. D 34, 1642 (1986). https://doi. org/10.1103/PhysRevD.34.1642

14. M. Malinsky, J. Romao, J. Valle, Phys. Rev. Lett. 95, 161801 (2005). https://doi.org/10.1103/PhysRevLett.95.161801. arXiv:0506296 [hep-ph]

15. P. B. Dev, R. Mohapatra, Phys. Rev. D 81, 013001 (2010). arXiv:0910.3924 [hep-ph]. https://doi.org/10.1103/PhysRevD. 81.013001

16. P. Dev, A. Pilaftsis, Phys. Rev. D 86, 113001 (2012). arXiv:1209.4051 [hep-ph]. https://doi.org/10.1103/PhysRevD. 86.113001

17. C.-H. Lee, P. Bhupal Dev, R. Mohapatra, Phys. Rev. D 88, 093010 (2013). arXiv:1309.0774 [hep-ph]. https://doi.org/10. 1103/PhysRevD.88.093010

18. W. Buchmüller, C. Greub, P. Minkowski, Phys. Lett. B 267, 395 (1991). https://doi.org/10.1016/0370-2693(91)90952-M

19. M. Kohda, H. Sugiyama, K. Tsumura, Phys. Lett. B 718, 1436 (2013). https://doi.org/10.1016/j.physletb.2012.12.048
20. T. Asaka, S. Blanchet, M. Shaposhnikov, Phys. Lett. B 631, 151 (2005). https://doi.org/10.1016/j.physletb.2005.09.070. arXiv:hep-ph/0503065 [hep-ph]

21. T. Asaka M. Shaposhnikov, Phys. Lett. B. 620, 17 (2005). arXiv:hep-ph/0505013 [hep-ph]. https://doi.org/10.1016/j. physletb.2005.06.020

22. F. del Aguila, J. Aguilar-Saavedra, J. de Blas, M. Zralek, Acta Phys. Polon. B 38, 3339 (2007). arXiv:0710.2923 [hep-ph]

23. X.-G. He, S. Oh, J. Tandean, C.-C. Wen, Phys. Rev. D 80, 073012 (2009). https://doi.org/10.1103/PhysRevD.80.073012. arXiv:0907.1607 [hep-ph]

24. J. Kersten, A.Y. Smirnov, Phys. Rev. D 76, 073005 (2007). arXiv:0705.3221 [hep-ph]. https://doi.org/10.1103/PhysRevD. 76.073005

25. A. Ibarra, E. Molinaro, S. Petcov, JHEP 09, 108 (2010). https:// doi.org/10.1007/JHEP09(2010)108. arXiv:1007.2378 [hep-ph]

26. M. Nemevšek, G. Senjanović, Y. Zhang, JCAP 07, 006 (2012). https://doi.org/10.1088/1475-7516/2012/07/006. arXiv:1205.0844 [hep-ph]

27. A. Donini, P. Hernandez, J. Lopez-Pavon, M. Maltoni, T. Schwetz, JHEP 07, 161 (2012). https://doi.org/10.1007/JHEP07(2012)161. arXiv: 1205.5230 [hep-ph]

28. G. Racah, Nuovo Cim. 14, 322 (1937). https://doi.org/10.1007/ BF02961321

29. W. Furry, Phys. Rev. 56, 1184 (1939). https://doi.org/10.1103/ PhysRev.56.1184

30. H. Primakoff, S. Rosen, Rept. Prog. Phys. 22, 121 (1959). https:// doi.org/10.1088/0034-4885/22/1/305

31. H. Primakoff S.P. Rosen, Phys. Rev. 184, 1925 (1969). https:// doi.org/10.1103/PhysRev.184.1925

32. H. Primakoff, P. S. Rosen, Ann. Rev. Nucl. Part. Sci. 31, 145 (1981). https://doi.org/10.1146/annurev.ns.31.120181.001045

33. J. Schechter, J. Valle, Phys. Rev. D 25, 2951 (1982). https://doi. org/10.1103/PhysRevD.25.2951

34. M. Doi, T. Kotani, E. Takasugi, Prog. Theor. Phys. Suppl. 83, 1 (1985). https://doi.org/10.1143/PTPS.83.1

35. S.R. Elliott, J. Engel, J. Phys. G 30, R183 (2004). arXiv:hep-ph/0405078. https://doi.org/10.1088/0954-3899/30/ 9/R01

36. V. Rodin, A. Faessler, F. Šimkovic, P. Vogel, Nucl. Phys. A 766, 107 (2006). [Erratum: Nucl.Phys.A 793, 213-215 (2007)]. arXiv:0706.4304 [nucl-th]. https://doi.org/10.1016/j.nuclphysa. 2005.12.004

37. L.S. Littenberg, R.E. Shrock, Phys. Rev. Lett. 68, 443. (1992). https://doi.org/10.1103/PhysRevLett.68.443

38. L.S. Littenberg R. Shrock, Phys. Lett. B 491, 285 (2000). arXiv:hep-ph/0005285. https://doi.org/10.1016/ S0370-2693(00)01041-8

39. C. Dib, V. Gribanov, S. Kovalenko, I. Schmidt, Phys. Lett. B 493 82 (2000). https://doi.org/10.1016/S0370-2693(00)01134-5. arXiv:hep-ph/0006277

40. A. Ali, A. Borisov, N. Zamorin, Eur. Phys. J. C 21, 123 (2001). https://doi.org/10.1007/s100520100702. arXiv:hep-ph/0104123

41. M.A. Ivanov, S.G. Kovalenko, Phys. Rev. D 71, 053004 (2005). arXiv:hep-ph/0412198. https://doi.org/10.1103/PhysRevD.71. 053004

42. A. de Gouvea, J. Jenkins, Phys. Rev. D 77, 013008 (2008). arXiv:0708.1344 [hep-ph]. https://doi.org/10.1103/PhysRevD. 77.013008

43. D. Delepine, G. López Castro, N. Quintero, Phys. Rev. D 84 (2011). https://doi.org/10.1103/physrevd.84.096011

44. G. López Castro N. Quintero, Phys. Rev. D 87 (2013). https://doi. org/10.1103/physrevd.87.077901

45. A. Abada, A. Teixeira, A. Vicente, C. Weiland, JHEP 02, 091 (2014). https://doi.org/10.1007/JHEP02(2014)091. arXiv:1311.2830 [hep-ph] 
46. Y. Wang, S.-S. Bao, Z.-H. Li, N. Zhu, Z.-G. Si, Phys. Lett. B 736, 428 (2014). https://doi.org/10.1016/j.physletb.2014.08.006

47. J.C. Helo, S. Kovalenko, I. Schmidt, Nucl. Phys. B 853, 80 (2011). https://doi.org/10.1016/j.nuclphysb.2011.07.020. arXiv: 1005.1607 [hep-ph]

48. A. Atre, T. Han, S. Pascoli, B. Zhang, JHEP 0905, 030 (2009). https://doi.org/10.1088/1126-6708/2009/05/030. arXiv:0901.3589 [hep-ph]

49. G. Cvetič, C. Dib, S.K. Kang, C.S. Kim, Phys. Rev. D 82, (2010). https://doi.org/10.1103/physrevd.82.053010

50. G. Cvetič, C. Dib, C.S. Kim, J. High Energy Phys. 2012, (2012). https://doi.org/10.1007/jhep06(2012)149

51. G. Cvetič, C.S. Kim, Z.-S. Jilberto, J. Phys. G: Nucl. Part. Phys. 41, 075004 (2014). https://doi.org/10.1088/0954-3899/41/7/075004

52. G. Cvetič, C. Dib, C.S. Kim, Z.-S. Jilberto, Symmetry 7, 726 (2015). https://doi.org/10.3390/sym7020726

53. D. Milanes, N. Quintero, C.E. Vera, Phys. Rev. D 93, 094026 (2016). https://doi.org/10.1103/PhysRevD.93.094026. arXiv:1604.03177 [hep-ph]

54. S. Mandal, N. Sinha, Phys. Rev. D 94 (2016). https://doi.org/10. 1103/physrevd.94.033001

55. G. Moreno, J. Zamora-Saá, Phys. Rev. D 94, 093005 (2016). arXiv:1606.08820 [hep-ph]. https://doi.org/10.1103/PhysRevD. 94.093005

56. V. Gribanov, S. Kovalenko, I. Schmidt, Nucl. Phys. B 607, 355 (2001). https://doi.org/10.1016/s0550-3213(01)00169-9

57. G. Cvetič, C. Dib, C.S. Kim, J.D. Kim, Phys. Rev. D 66, 034008 (2002). [Erratum: Phys. Rev. D 68, 059901 (2003)], arXiv:hep-ph/0202212. https://doi.org/10.1103/PhysRevD.66. 034008

58. J.C. Helo, S. Kovalenko, I. Schmidt, Phys. Rev. D 84 (2011). https://doi.org/10.1103/physrevd.84.053008

59. J. Zamora-Saá, J. High Energy Phys. 2017, (2017). https://doi. org/10.1007/jhep05(2017)110

60. S. Tapia, J. Zamora-Saá, Nucl. Phys. B 952, 114936 (2020). arXiv:1906.09470 [hep-ph]. https://doi.org/10.1016/j.nuclphysb. 2020.114936

61. W.-Y. Keung, G. Senjanović, Phys. Rev. Lett. 50, 1427 (1983). https://doi.org/10.1103/PhysRevLett.50.1427

62. V. Tello, M. Nemevšek, F. Nesti, G. Senjanović, F. Vissani, Phys. Rev. Lett. 106, (2011). https://doi.org/10.1103/physrevlett.106. 151801

63. M. Nemevšek, F. Nesti, G. Senjanović, V. Tello, Neutrinoless double beta decay: low left-right symmetry scale? (2011). arXiv:1112.3061 [hep-ph]

64. S. Kovalenko, Z. Lu, I. Schmidt, Phys. Rev. D 80, (2009). https:// doi.org/10.1103/physrevd.80.073014

65. C.-Y. Chen, P. S. B. Dev, Phys. Rev. D 85 (2011). https://doi.org/ 10.1103/physrevd.85.093018

66. C.-Y. Chen, P.S.B. Dev, R.N. Mohapatra, Phys. Rev. D 88, (2013). https://doi.org/10.1103/physrevd.88.033014

67. P.B. Dev, A. Pilaftsis, U.-K. Yang, Phys. Rev. Lett. 112, (2014). https://doi.org/10.1103/physrevlett.112.081801

68. A. Das, N. Okada, Phys. Rev. D 88 (2013). https://doi.org/10. 1103/physrevd.88.113001

69. A. Das, P. Bhupal Dev, N. Okada, Phys. Lett. B 735, 364 (2014). arXiv:1405.0177 [hep-ph]. https://doi.org/10.1016/ j.physletb.2014.06.058

70. D. Alva, T. Han, R. Ruiz, J. High Energy Phys. 2015, (2015). https://doi.org/10.1007/jhep02(2015)072

71. A. Das, N. Okada, Phys. Rev. D 93 (2016). https://doi.org/10. 1103/physrevd.93.033003

72. A. Das, N. Okada, Phys. Lett. B 774, 32 (2017). https://doi.org/ 10.1016/j.physletb.2017.09.042

73. C. Degrande, O. Mattelaer, R. Ruiz, J. Turner, Phys. Rev. D 94, (2016). https://doi.org/10.1103/physrevd.94.053002
74. A. Das, P. Konar, S. Majhi, J. High Energy Phys. 2016, (2016). https://doi.org/10.1007/jhep06(2016)019

75. A. Das (2017). arXiv:1701.04946 [hep-ph]

76. W. Buchmüller, C. Greub, Nucl. Phys. B 363, 345 (1991). https:// doi.org/10.1016/0550-3213(91)80024-G

77. J. Helo, S. Kovalenko, M. Hirsch, Phys. Rev. D 89, (2014). https:// doi.org/10.1103/physrevd.89.073005

78. C. O. Dib C. S. Kim, Phys. Rev. D 92 (2015). https://doi.org/10. 1103/physrevd.92.093009

79. C.O. Dib, C.S. Kim, K. Wang, J. Zhang, Phys. Rev. D 94, (2016). https://doi.org/10.1103/physrevd.94.013005

80. C.O. Dib, C.S. Kim, K. Wang, Phys. Rev. D 95, (2017). https:// doi.org/10.1103/physrevd.95.115020

81. C.O. Dib, C.S. Kim, K. Wang, Chin. Phys. C 41, 103103 (2017). https://doi.org/10.1088/1674-1137/41/10/103103

82. A. Das, P.S.B. Dev, C.S. Kim, Phys. Rev. D 95, (2017). https:// doi.org/10.1103/physrevd.95.115013

83. A. Das, Y. Gao, T. Kamon, Eur. Phys. J. C 79, (2019). https://doi. org/10.1140/epjc/s10052-019-6937-7

84. G. 't Hooft, Phys. Rev. Lett. 37, 8 ( 1976). https://doi.org/10.1103/ PhysRevLett.37.8

85. G. 't Hooft, Phys. Rev. D 14, 3432 (1976). [Erratum: Phys. Rev. D 18, 2199 (1978)] https://doi.org/10.1103/PhysRevD.14.3432

86. P. Ade et al. (Planck), Astron. Astrophys. 594, A13 (2016). arXiv:1502.01589 [astro-ph.CO]. https://doi.org/10. 1051/0004-6361/201525830

87. A.D. Sakharov, Phys. Usp. 34, 392 (1991). https://doi.org/10 1070/PU1991v034n05ABEH002497

88. M. Fukugita T. Yanagida, Phys. Lett. B 174, 45 (1986). https:// doi.org/10.1016/0370-2693(86)91126-3

89. W. Buchmüller, R. Peccei, T. Yanagida, Ann. Rev. Nucl. Part. Sci. 55, 311 (2005). https://doi.org/10.1146/annurev.nucl.55.090704. 151558. arXiv:hep-ph/0502169

90. W. Buchmüller, P. Di Bari, M. Plumacher, Ann. Phys. 315, 305 (2005). https://doi.org/10.1016/j.aop.2004.02.003. arXiv:hep-ph/0401240

91. E.K. Akhmedov, V. Rubakov, A. Smirnov, Phys. Rev. Lett. 81, 1359 (1998). https://doi.org/10.1103/PhysRevLett.81.1359. arXiv:hep-ph/9803255

92. S. Davidson A. Ibarra, Phys. Lett. B 535, 25 (2002). arXiv:hep-ph/0202239. https://doi.org/10.1016/ S0370-2693(02)01735-5

93. A. Pilaftsis T. E. Underwood, Nucl. Phys. B 692, 303 (2004). arXiv:hep-ph/0309342. https://doi.org/10.1016/j.nuclphysb. 2004.05.029

94. E.J. Chun et al., Int. J. Mod. Phys. A 33, 1842005 (2018). https:// doi.org/10.1142/S0217751X18420058. arXiv:1711.02865 [hep$\mathrm{ph}]$

95. A. Pilaftsis, Phys. Rev. D 56, 5431 (1997). https://doi.org/10. 1103/PhysRevD.56.5431. arXiv:hep-ph/9707235 [hep-ph]

96. S. Bray, J.S. Lee, A. Pilaftsis, Nucl. Phys. B 786, 95 (2007). https://doi.org/10.1016/j.nuclphysb.2007.07.002. arXiv:hep-ph/0702294 [HEP-PH]

97. P. Hernadez, J. Jones-Perez, O. Suarez-Navarro, Eur. Phys. J. C 79, 220 (2019). https://doi.org/10.1140/epjc/s10052-019-6728-1. arXiv: 1810.07210 [hep-ph]

98. G. Cvetič, C.S. Kim, J. Zamora-Saá, Phys. Rev. D 89, 093012 (2014). https://doi.org/10.1103/PhysRevD.89.093012. arXiv: 1403.2555 [hep-ph]

99. C.O. Dib, M. Campos, C.S. Kim, JHEP 1502, 108 (2015). https:// doi.org/10.1007/JHEP02(2015)108. arXiv:1403.8009 [hep-ph]

100. G. Cvetič, C. Dib, C.S. Kim, J. Zamora-Saa, Symmetry 7, 726 (2015). arXiv:1503.01358 [hep-ph]

101. G. Cvetič, C.S. Kim, J. Zamora-Saá, J. Phys. G41, 075004 (2014). https://doi.org/10.1088/0954-3899/41/7/075004. arXiv:1311.7554 [hep-ph] 
102. A. Abada, C. Hati, X. Marcano, A. Teixeira, JHEP 09, 017 (2019). https://doi.org/10.1007/JHEP09(2019)017. arXiv:1904.05367 [hep-ph]

103. G. Cvetič, C.S. Kim, R. Kögerler, J. Zamora-Saá, Phys. Rev. D 92, 013015 (2015). https://doi.org/10.1103/PhysRevD.92. 013015. arXiv:1505.04749 [hep-ph]

104. G. Cvetič, A. Das, J. Zamora-Saá, J. Phys. G46, 075002 (2019). https://doi.org/10.1088/1361-6471/ab1212. arXiv:1805.00070 [hep-ph]

105. G. Cvetič, A. Das, S. Tapia, J. Zamora-Saá, J. Phys. G 47, 015001 (2020). https://doi.org/10.1088/1361-6471/ab4ee8. arXiv:1905.03097 [hep-ph]

106. J. Zamora-Saá, JHEP 05, 110 (2017). https://doi.org/10.1007/ JHEP05(2017)110. arXiv:1612.07656 [hep-ph]

107. G. Anamiati, M. Hirsch, E. Nardi, JHEP 10, 010 (2016). https:// doi.org/10.1007/JHEP10(2016)010. arXiv:1607.05641 [hep-ph]

108. S. Antusch, E. Cazzato, O. Fischer, Mod. Phys. Lett. A 34, 1950061 (2019). https://doi.org/10.1142/S0217732319500615. arXiv:1709.03797 [hep-ph]

109. A. Das, P.S.B. Dev, R.N. Mohapatra, Phys. Rev. D 97, 015018 (2018). https://doi.org/10.1103/PhysRevD.97.015018. arXiv:1709.06553 [hep-ph]

110. G. Cvetič C. S. Kim, Phys. Rev. D 94, 053001 (2016). [Erratum: Phys. Rev. D 95, 039901 (2017)]. arXiv:1606.04140 [hep-ph]. https://doi.org/10.1103/PhysRevD.94.053001
111. G. Cvetič C. Kim, Phys. Rev. D 96, 035025 (2017). [Erratum: Phys. Rev. D 102, 039902 (2020)]. arXiv:1705.09403 [hep-ph]. https://doi.org/10.1103/PhysRevD.96.035025

112. L. Duarte, J. Peressutti, I. Romero, O.A. Sampayo, Eur. Phys. J. C 79, 593 (2019). https://doi.org/10.1140/epjc/ s10052-019-7104-x. arXiv:1904.07175 [hep-ph]

113. L. Duarte, G. Zapata, O. Sampayo, (2020). arXiv:2006.11216 [hep-ph]

114. G.P. Lepage, J. Comput. Phys. 27, 192 (1978). https://doi.org/10. 1016/0021-9991(78)90004-9

115. A. Abada, V. De Romeri, M. Lucente, A.M. Teixeira, T. Toma, JHEP 02, 169 (2018). https://doi.org/10.1007/JHEP02(2018)169. arXiv:1712.03984 [hep-ph]

116. W. Altmannshofer et al. (Belle-II), PTEP 2019, 123 C01 (2019). [Erratum: PTEP 2020, 029201 (2020)]. arXiv:1808.10567 [hepex]. https://doi.org/10.1093/ptep/ptz106

117. I. Caprini, L. Lellouch, M. Neubert, Nucl. Phys. B 530, 153 (1998). https://doi.org/10.1016/S0550-3213(98)00350-2. arXiv:hep-ph/9712417

118. R. Glattauer et al. (Belle), Phys. Rev. D 93, 032006 (2016). arXiv:1510.03657 [hep-ex]. https://doi.org/10.1103/PhysRevD. 93.032006 